\title{
Turin-SyCAT: A multifrequency catalog of Seyfert galaxies ${ }^{\star}$
}

\author{
H. A. Peña-Herazo ${ }^{1,2,3}$, F. Massaro ${ }^{1,4,5,6} \odot$, V. Chavushyan ${ }^{2} \odot$, N. Masetti ${ }^{7,8} \odot$, A. Paggi $^{1,4,5} \odot$, and A. Capetti ${ }^{5}$ \\ 1 Dipartimento di Fisica, Università degli Studi di Torino, Via Pietro Giuria 1, 10125 Torino, Italy \\ e-mail: h.pena@eaobservatory.org \\ 2 Instituto Nacional de Astrofísica, Óptica y Electrónica, Apartado Postal 51-216, 72000 Puebla, Mexico \\ 3 East Asian Observatory, 660 North A'ohōkū Place, Hilo, Hawaii 96720, USA \\ ${ }^{4}$ Istituto Nazionale di Fisica Nucleare, Sezione di Torino, 10125 Torino, Italy \\ 5 INAF-Osservatorio Astrofisico di Torino, Via Osservatorio 20, 10025 Pino Torinese, Italy \\ ${ }^{6}$ Consorzio Interuniversitario per la Fisica Spaziale (CIFS), Via Pietro Giuria 1, 10125 Torino, Italy \\ 7 INAF - Osservatorio di Astrofisica e Scienza dello Spazio, Via Gobetti 93/3, 40129 Bologna, Italy \\ 8 Departamento de Ciencias Físicas, Universidad Andrés Bello, Fernández Concha 700, Las Condes, Santiago, Chile
}

Received 25 June 2020 / Accepted 10 September 2021

\begin{abstract}
Aims. We present the first release of Turin-SyCAT, a multifrequency catalog of Seyfert galaxies.

Methods. We selected Seyfert galaxies considering criteria based on radio, infrared, and optical properties and starting from sources belonging to hard X-ray catalogs and surveys. We visually inspected optical spectra available for all selected sources. We adopted homogeneous and stringent criteria in the source selection aiming at reducing the possible contamination from other source classes. Results. Our final catalog includes 351 Seyfert galaxies distinguished in 233 type 1 and 118 type 2. Type 1 Seyfert galaxies appear to have mid-IR colors similar to blazars, but are distinguished from them by their radio-loudness. Additionally, Seyfert 2 galaxies have mid-IR colors more similar to quasars than BL Lac objects. As expected from their spectral properties, type 1 and 2 Seyfert galaxies show a clear distinction when using the $u-r$ color. Finally, we found a tight correlation between the mid-IR fluxes at both 12 and $22 \mu \mathrm{m}$ (i.e., $F_{12}$ and $F_{22}$, respectively) and hard X-ray fluxes between 15 and $150 \mathrm{keV}$. Both Seyfert types appear to follow the same trend and share similar values of the ratios of $F_{12}$ and $F_{22}$ to $F_{H X}$ in agreement with expectations of the AGN unification scenario. Conclusions. As future perspectives, the Turin-SyCAT will then be used to search for heavily obscured Seyfert galaxies among unidentified hard X-ray sources, given the correlation between mid-IR and hard X-rays, and to investigate their large-scale environments.
\end{abstract}

Key words. galaxies: Seyfert - catalogs - galaxies: active

\section{A brief historical introduction}

The story of Seyfert galaxies began in 1908 when Edward A. Fath discovered peculiar emission lines in the nuclear spectrum of the spiral nebula NGC 1068 (Fath 1909) at the Lick Observatory, later confirmed by Vesto M. Slipher in 1917 (Slipher 1917). At that epoch, the largest fraction of known extragalactic sources showed an optical spectrum dominated by absorption features due to stars, while NGC 1068 presented several relatively bright emission lines.

About two decades later, Hubble (1926), Humason (1932), and Mayall (1934) discovered similar optical emission lines in other nebulae, then identifying them as extragalactic sources. However, we had to wait until 1943 when Carl Keenan Seyfert, reporting on his study of five more galaxies similar to NGC 1068 (namely NGC 1275, 3516, 4051, 4151, and 7469), established the first class of active galactic nuclei (AGNs), showing emission dominated by their nuclear regions. At the end of the 1950s, the "Seyfert galaxies" were mainly characterized by extremely compact nuclei (i.e., $<100 \mathrm{pc}$ ) with masses on the order of $10^{8}-10^{9} M_{\odot}$ coupled with emission lines indicating velocities

* Full Tables A.1-A.5 are only available at the CDS via anonymous ftp to cdsarc.u-strasbg.fr $(130.79 .128 .5)$ or via http: //cdsarc.u-strasbg.fr/viz-bin/cat/J/A+A/659/A32 of few thousand kilometers per second in their optical spectra (Burbidge et al. 1959; Woltjer 1959).

In the early 1960s, after the discovery of quasars as cosmological sources (Schmidt 1963), it became clear that Seyfert galaxies shared several properties with them, certainly their cosmological distances (i.e., redshift $z$ ), but were less luminous. As more Seyfert galaxies and quasars were discovered, their separation in luminosity narrowed down and almost overlapped (see also Green 1976; Burbidge et al. 1963; Osterbrock \& Parker 1965; Pacholczyk \& Weymann 1968; Burbidge \& Hoyle 1968; Weedman 1976; Schmidt \& Green 1983). At that time the community also started noticing that Seyfert galaxies were preferentially hosted in spiral galaxies (Markarian 1967; Sargent 1970; Green 1976; Adams 1977; Heckman 1978; Simkin et al. 1980; Yee 1983; MacKenty 1990; Kotilainen \& Ward 1994; Xanthopoulos 1996).

A major step forward for understanding this new class of extragalactic sources was carried out in 1974, when Khachikian and Weedman identified two types of Seyfert galaxies. Optical spectra of Seyfert galaxies are mainly characterized by the presence of permitted emission lines, such as Balmer lines, $\mathrm{He}$ II $\lambda 4686$ and $\mathrm{He} \mathrm{I} \lambda 5876, \mathrm{Fe}$ II, and forbidden emission lines: [O II] $\lambda 3727$, [O III] $\lambda \lambda 4959,5007$, [S II] $\lambda \lambda 6717,6731$, and [N II] $\lambda \lambda 6548,6584$. Balmer lines can have broad $(F W H M>$ $10^{3} \mathrm{~km} \mathrm{~s}^{-1}$ ) and narrow components in their profiles. Thus, 
Seyfert galaxies were classified initially as type 1 or 2 based on the relative width of their Balmer or forbidden emission lines (Khachikian \& Weedman 1971, 1974). Type 2 are those with a relative width of $\mathrm{H} \beta$ that is similar to that of the [OIII] lines (Weedman 1977; Ho 2008).

Furthermore, Osterbrock (1981) introduced a deeper qualitative classification to account for the range of relative strengths of the $\mathrm{H} \alpha$ and $\mathrm{H} \beta$ broad components compared with the narrow lines. Intermediate types of Seyfert galaxies were defined using the broad component of these emission lines, being weaker as indicated by their classification numbers: $1.2,1.5,1.8$, and 1.9. In the last case the broad component is detectable in $\mathrm{H} \alpha$ but not in $\mathrm{H} \beta$.

Seyfert galaxies can be distinguished from other types of emission-line galaxies, for example starburst and star-forming galaxies or low-ionization nuclear emission-line regions (LINERs) by using diagnostic diagrams based on the line intensity ratios (Veilleux \& Osterbrock 1987; Kewley et al. 2006; Stasińska et al. 2006). One of the most used diagnostic tools is the Baldwin-Phillips-Terlevich (BPT) diagram (Baldwin et al. 1981) based on the comparison between the intensity ratios of the [O III] $\lambda 5007$ to $\mathrm{H} \beta$ emission lines and of the [N II] $\lambda 6584$ to $\mathrm{H} \alpha$ emission lines, where AGNs are well separated from starforming galaxies (see also Ho et al. 1997; Kewley et al. 2001, 2006; Kauffmann et al. 2003). It is worth noting that in the BPT diagram Seyfert galaxies and LINERs tend to lie in the same region, both having relative intense low-ionization lines (e.g.,

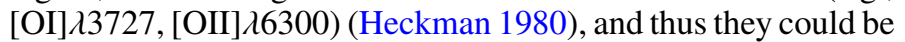
eventually distinguished according to some empirical criteria, as outlined by Schawinski et al. (2007).

At other wavelengths Seyfert galaxies are commonly known as radio-quiet sources, with radio power typically less than $\sim 10^{40} \mathrm{erg} \mathrm{s}^{-1}$ (Ho et al. 1995; Ho \& Ulvestad 2001). Radio emission in Seyfert galaxies (see, e.g., Panessa et al. 2019; Chiaraluce et al. 2019, for recent reviews) shows compact morphology and flat spectrum, and extended parsec-scale emission with brightness temperature $>10^{7} \mathrm{~K}$ resembling jet emission, as shown by large baseline interferometry (see also Bontempi et al. 2012; Sebastian et al. 2020).

Seyfert galaxy emission at infrared (IR) frequencies accounts for a significant percentage of their bolometric luminosity. Their IR light consists of several components, including continuum emission dominated by the re-processed radiation arising from the dusty torus, heated by the central AGN (Efstathiou \& Rowan-Robinson 1995; Tristram et al. 2007; Nenkova et al. 2008; Mor et al. 2009; Alonso-Herrero et al. 2011; Siebenmorgen et al. 2015; González-Martín et al. 2019). In addition, their IR emission also show (i) a contribution due to star formation (Cid Fernandes et al. 2001; Davies et al. 2007; LaMassa et al. 2012; Diamond-Stanic \& Rieke 2012; García-Bernete et al. 2016; Ruschel-Dutra et al. 2017; Esparza-Arredondo et al. 2018); (ii) polycyclic aromatic hydrocarbons, again related with star formation (Peeters et al. 2004; Sani et al. 2010; Gallimore et al. 2010; Calzetti 2011; Jensen et al. 2017); (iii) emission lines from fine structure ionic species (e.g., [Ne II] $12.8 \mu \mathrm{m},[\mathrm{Ne}$ III] $15.5 \mu \mathrm{m},[\mathrm{Ne}$ V] $14.5 \mu \mathrm{m}$, [S IV] $10.5 \mu \mathrm{m}$ ) (Ruschel-Dutra et al. 2014), and silicate emissions or other molecular emission (Sturm et al. 2005; Hao et al. 2007; Roche et al. 2007; Mason et al. 2009; Gallimore et al. 2010; Xie et al. 2017; Menezes et al. 2018), often associated with outflows (see, e.g., García-Burillo et al. 2014; Morganti et al. 2015; Alonso-Herrero et al. 2019; Feruglio et al. 2020).

At high energies Seyfert galaxies are also detected in the Xray band with luminosities $L_{2-10 \mathrm{keV}} \sim 10^{38-43} \mathrm{erg} \mathrm{s}^{-1}$ (see, e.g.,
Panessa et al. 2006, for recent work on XMM-Newton and Chandra satellites, and references therein). Their soft X-ray spectra are dominated by a power-law continuum, coupled with a high-energy cutoff, both due to inverse Compton of low-energy photons, from the accretion disk, on relativistic electrons of a hot corona (see, e.g., Mushotzky et al. 1980; Haardt \& Maraschi 1991; Singh et al. 2011; Bianchi et al. 2009a). In addition to this X-ray continuum, below a few $\mathrm{keV}$ there is a featureless soft excess mainly due to unresolved X-ray emission lines (Fabian et al. 1986; Walter \& Fink 1993; Mehdipour et al. 2011; Gliozzi \& Williams 2020; Massaro et al. 2006; Bianchi et al. $2006,2009 b$ ), while above $\sim 6 \mathrm{keV}$ the presence of the Fe K $\alpha$ fluorescent emission line is often evident, mainly arising from the reflection of the coronal X-ray emission on the accretion disk and the dusty torus (George \& Fabian 1991; Nandra \& Pounds 1994; Matt et al. 1996; Bianchi et al. 2004; Markowitz et al. 2008; Patrick et al. 2012; Mantovani et al. 2016).

In X-rays, type 1 and 2 Seyfert galaxies are often distinguished on the basis of their intrinsic absorbing column density, with type 2 having $N_{H \text {,int }}>10^{22} \mathrm{~cm}^{-2}$. However, there are heavily absorbed Seyfert 1 galaxies mismatching their optical classifications (Panessa \& Bassani 2002; Tueller et al. 2008; Beckmann et al. 2009; Corral et al. 2011; Merloni et al. 2014; Ordovás-Pascual et al. 2017). Considering Seyfert 1.8-1.9 to be optically unobscured, Burtscher et al. (2016) suggest that $N_{H \text {,int }}>10^{22.3} \mathrm{~cm}^{-2}$ matches the X-ray versus optical classification. Moreover, local Seyfert 2 galaxies, selected by [O III] flux, show intrinsic column densities $N_{H}>10^{24} \mathrm{~cm}^{-2}$ (Maiolino et al. 1998; Risaliti et al. 1999; Ricci et al. 2015), thus are classified as Compton-thick (Matt 1997). In the literature there are reported cases of Compton-thick Seyfert galaxies that changed to Compton-thin according to their X-ray spectral evolution (see, e.g., Matt et al. 2003). Then, at higher energies Seyfert galaxies in the local Universe are also detected in $\gamma$ rays, possibly coupled with the starburst activity (Hayashida et al. 2013; Ackermann et al. 2012; Wojaczyński et al. 2015).

At zero order, according to the unification model of AGNs (Antonucci 1993), Seyfert galaxies of type 1 and 2 are intrinsically the same but viewed at different angles with respect to the line of sight. In particular, Seyfert 2 galaxies are those observed at larger viewing angles, thus having the central engine obscured by the presence of a dusty torus (see also Pogge 1988). Evidence regarding the role of orientation was found early on by Keel (1980), showing that Seyfert 2 galaxies have random orientation, while Seyfert 1 galaxies are predominantly orientated faceon. This was later confirmed when Antonucci \& Miller (1985) found a hidden Seyfert 1-like spectrum in the polarized light of classical Seyfert 2 NGC 1068.

However, today the whole picture appears more complicated since several discoveries are challenging the unification scenario. Observational evidence provided by (i) changing look Seyfert galaxies (e.g., Collin-Souffrin et al. 1973; Aretxaga et al. 1999; LaMassa et al. 2015; Denney et al. 2014; Shappee et al. 2014; Raimundo et al. 2019), (ii) Seyfert 2 galaxies without absorption in X-rays (Pappa et al. 2001), (iii) rare Seyfert galaxies lacking the broad-line region (Panessa \& Bassani 2002; Shi et al. 2010), and (iv) the role of the environment triggering the AGN activity (see, e.g., De Robertis et al. 1998; Koulouridis et al. 2006; Villarroel \& Korn 2014) are not perfectly in agreement with the previous statements. Then additional analyses of the large-scale environments of Seyfert galaxies discovered a significant excess in the number of companions compared with non-active galaxies (Petrosian 1982; Dahari 1984; MacKenty 1989; Laurikainen \& Salo 1995; 
Rafanelli et al. 1995; Salvato \& Rafanelli 1997), and other investigations also found a difference between the ambient richness of the Seyfert 1 and 2 classes, the latter found in environments with higher galaxy density (Dultzin-Hacyan et al. 1999; Koulouridis et al. 2006; Jiang et al. 2016; Villarroel \& Korn 2014; Gordon et al. 2017).

In the literature several catalogs or samples of Seyfert galaxies already exist. One of the most statistically robust samples, selected on the basis of homogeneous criteria, is certainly the one based on the Palomar spectroscopic survey of nearby galaxies (Ho et al. 1997, 2003), but unfortunately it lists only 52 Seyfert galaxies. Other Seyfert galaxy catalogs or samples present in the literature are those of Lipovetsky et al. (1987) and Lipovetsky et al. (1988), composed of 959 sources, most of the Seyfert galaxies known at that time, selected via the UV excess method drawn by the First Byurakan Survey (Markarian et al. 1989); the 49 Seyfert galaxies selected from the Harvard Center for Astrophysics (CfA) spectroscopic survey (Huchra \& Burg 1992); 80 type 1 and 141 type 2 Seyfert galaxies with observed spectra selected using far-IR colors (de Grijp et al. 1992); 91 Seyfert galaxies listed in the Maiolino \& Rieke (1995) sample based on the Revised Shapley-Ames catalog (Sandage \& Tammann 1987); 539 Seyfert galaxies present in the BASS survey (Koss et al. 2017); more than 16000 Seyfert galaxies listed in the catalog of quasars and active nuclei of Veron-Cetty \& Veron (1989), and Véron-Cetty \& Véron $(2006,2010)$ compiled including those AGN lists collected from an extensive literature search based on such surveys as 2dF/2QZ (Croom et al. 2004), SDSS DR-3 (Fukugita et al. 1996; Abazajian et al. 2005), as well as compact radio sources in the ICRF2 (Fey et al. 2015), VLBA (Beasley et al. 2002; Fomalont et al. 2003), the large quasar astrometric catalog (Souchay et al. 2009), and X-ray discovered AGNs (Treister et al. 2005). However, all these catalogs or samples are characterized either by having a relatively small number of sources (i.e., fewer than 100 objects) or being highly contaminated by different AGN classes, such as radio galaxies or quasars. In many cases sources listed in these samples lack optical spectroscopic redshifts, and Seyfert classification is not based on multifrequency criteria.

Here we present the first release of the Turin-SyCAT, a multifrequency selected catalog of Seyfert galaxies. Datasets, observations, and information regarding all sources listed therein are mainly gathered from radio, optical, infrared, and X-ray surveys and will permit searches for trends or correlations between different observed quantities (see, e.g., Stern et al. 2012; Gandhi et al. 2009; Ichikawa et al. 2019) and, as a future perspective, studies of the large-scale environment of Seyfert galaxies (see, e.g., Koulouridis et al. 2006; Jiang et al. 2016).

It is important to note that to build the Turin-SyCAT we adopted homogeneous and stringent selection criteria based on multifrequency observations, thus focusing on a source selection that minimizes the possible contamination of other source classes. We anticipate that this choice prevents us from claiming that the catalog is complete at any level.

An example of a catalog that inspired our choice is the Roma-BZCAT (Massaro et al. 2009, 2015a), which lists extragalactic sources known as blazars. The Roma-BZCAT was compiled using different surveys, catalogs, and lists of sources classified as blazars found in the literature. The Roma-BZCAT is undoubtedly incomplete and includes many different biases due to the selection criteria of the catalogs used to create it. However, the underlying, precise inspection of multifrequency observations available for all sources listed there combined with stringent classification criteria lead to an extremely low contamination from sources belonging to other classes.

Thanks to the homogenous and stringent criteria adopted for source selection of the Roma-BZCAT it was possible to discover the WISE $\gamma$-rays strip (Massaro et al. 2011), leading to new methods for searching for potential counterparts of FermiLAT unidentified or unassociated sources (e.g., Abdollahi et al. 2020); to study the luminosity function of blazars (e.g., Ajello et al. 2014); to select potential targets for the Cherenkov Telescope Array (CTA) (Arsioli et al. 2015); to search for counterparts of new flaring $\gamma$-ray sources (e.g., Bernieri et al. 2013); and to obtain limits on the dark matter annihilation in sub-halos (e.g., Zechlin \& Horns 2012; Berlin \& Hooper 2014). Thus, motivated by the experience collected thanks to the use of the Roma-BZCAT, we decided to build the Turin-SyCAT favoring a source selection with low contamination from other classes rather than its completeness.

This paper is organized as follows. In Sect. 2 we list all the catalogs and surveys used to carry out our initial Seyfert galaxy selection. Section 3 is devoted to the outline of all selection criteria and thresholds adopted. Section 4 is dedicated to the catalog description and cross-matches with multifrequency databases. Results of the characterization of the Seyfert galaxy population achieved thanks to this first release of the Turin-SyCAT is presented in Sect. 5. Section 6 presents our summary, conclusions, and future perspectives. All tables can be found in Appendix A. Finally, Appendix B is dedicated to checking redshift dependences in the WISE color-color space.

We use cgs units unless stated otherwise. WISE magnitudes are in the Vega system and are not corrected for the Galactic extinction. As shown in our previous analyses (D'Abrusco et al. 2014, 2019; Massaro \& D'Abrusco 2016), this correction affects mainly the magnitude at $3.4 \mu \mathrm{m}$ for sources lying at low Galactic latitudes (i.e., $|b|<20^{\circ}$ ), and it ranges between $2 \%$ and $5 \%$ of their magnitude values, thus do not significantly affect our results. We indicate the WISE magnitudes at 3.4, 4.6, 12, and $22 \mu \mathrm{m}$ as $\mathrm{W} 1, \mathrm{~W} 2, \mathrm{~W} 3$, and $\mathrm{W} 4$, respectively. For all WISE magnitudes of sources flagged as extended in the AllWISE catalog (i.e., extended flag "ext_flg" greater than 0) we used values measured in the elliptical aperture ${ }^{1}$. Sloan Digital Sky Survey (SDSS) and Panoramic Survey Telescope \& Rapid Response System (Pan-STARRS) magnitudes are in the AB system. We adopted $\Lambda$ CDM cosmology with $\Omega_{m}=0.286$, and Hubble constant $H_{0}=69.6 \mathrm{~km} \mathrm{~s}^{-1} \mathrm{Mpc}^{-1}$ (Bennett et al. 2014) to compute cosmological corrections. Finally, given the large number of acronyms used here, mostly due to the different classifications and telescopes used, we summarize them in Table 1.

\section{Surveys and catalogs}

We selected Seyfert galaxies for this first release of the TurinSyCAT from the following two main hard X-ray surveys.

- The third release of the Palermo BAT source catalog (hereafter 3 PBC) $)^{2}$, including all hard X-ray sources detected by the BAT instrument on board the Neil Gehrels Swift Observatory within the first 66 months of mission (see, e.g., Segreto et al. 2010; Cusumano et al. 2010).

The 3PBC includes 1586 sources, 1387 with an assigned low-energy counterpart, all detected above a level of confidence of $4.8 \sigma$ Gaussian equivalent. The survey has a

\footnotetext{
1 https://wise2.ipac.caltech.edu/docs/release/allwise/ expsup/sec2_1a.html

2 bat.ifc.inaf.it/bat_catalog_web/66m_bat_catalog.html
} 
Table 1. Acronyms used in the text.

\begin{tabular}{ll}
\hline \hline Acronym & Description \\
\hline AGN & Active galactic nuclei \\
LINER & $\begin{array}{l}\text { Low-ionization nuclear emission-line } \\
\text { region }\end{array}$ \\
\hline BAT & Burst alert telescope \\
INTEGRAL & $\begin{array}{l}\text { International gamma-ray astrophysics lab- } \\
\text { oratory }\end{array}$ \\
IRAS & Infrared astronomical satellite \\
Pan-STARRS & Panoramic survey telescope and rapid \\
& response system \\
ROSAT & Roentgen satellite \\
SWIFT & Neil Gehrels Swift Observatory \\
WISE & Wide-field infrared survey explorer \\
\hline 3C & Third cambridge catalog \\
4C & Fourth cambridge catalog \\
3PBC & Third Palermo BAT catalog \\
BAT105 & Swift-BAT 105 month catalog \\
IBISCAT4 & 4th IBIS/ISGRI soft gamma-ray survey \\
& catalog \\
RBSC & ROSAT bright source catalog \\
FRSC & ROSAT faint source catalog \\
UHX & Unidentified hard X-ray sources \\
\hline 2MASS & Two micron all-sky survey \\
6dFG & Six-degree field galaxy survey \\
BASS & BAT AGN spectroscopic survey \\
NVSS & NRAO very large array sky survey \\
SDSS & Sloan digital sky survey \\
SUMSS & Sydney university Molonglo sky survey \\
\hline KDE & Kernel density estimation \\
EW & Equivalent width \\
FWHM & Full width at half maximum \\
S/N & Signal-to-noise ratio \\
\hline & \\
\hline
\end{tabular}

flux limit of $9.2 \times 10^{-12} \mathrm{erg} \mathrm{s}^{-1} \mathrm{~cm}^{-2}$ at Galactic latitudes $|b|>10^{\circ}$. For the construction of 3PBC Cusumano et al. (2010) used a dedicated procedure presented with details in Segreto et al. (2010). This procedure optimizes the signalto-noise ratio in energy and time; it performs screening, mosaicking, and source detection on masked data in several energy bands, namely $15-30 \mathrm{keV}, 15-70 \mathrm{keV}$ and $15-$ $150 \mathrm{keV}$. Then 3PBC counterparts were associated by crosscorrelating with source databases and analyzing imaging data from soft X-ray datasets, such as those collected from Swift-XRT.

- The BAT 105-month survey (hereafter BAT105) $)^{3}$ carried out again using the data collected by the BAT instrument, but reduced with a different procedure with respect to that of the 3PBC and by a different group (Oh et al. 2018). The BAT105 hard X-ray catalog is based on a 105-month Swift-BAT allsky hard X-ray survey listing 1632 sources, of which 129 unidentified sources in 14-195 keV energy band again above $4.8 \sigma$ Gaussian equivalent detection significance. The survey has a flux limit of $8.4 \times 10^{-12} \mathrm{erg} \mathrm{s}^{-1} \mathrm{~cm}^{-2}$ over $90 \%$ of the sky. To build the BAT105, Oh et al. (2018) extracted data from single snapshot images in the energy bands 14$20 \mathrm{keV}, 20-24 \mathrm{keV}, 24-35 \mathrm{keV}, 35-50 \mathrm{keV}, 50-75 \mathrm{keV}, 75-$ $100 \mathrm{keV}, 100-150 \mathrm{keV}$, and $150-195 \mathrm{keV}$, and then built allsky mosaics and band-integrated images. BAT105 coun-

3 swift.gsfc.nasa.gov/results/bs 105 mon
3PBC identification fraction

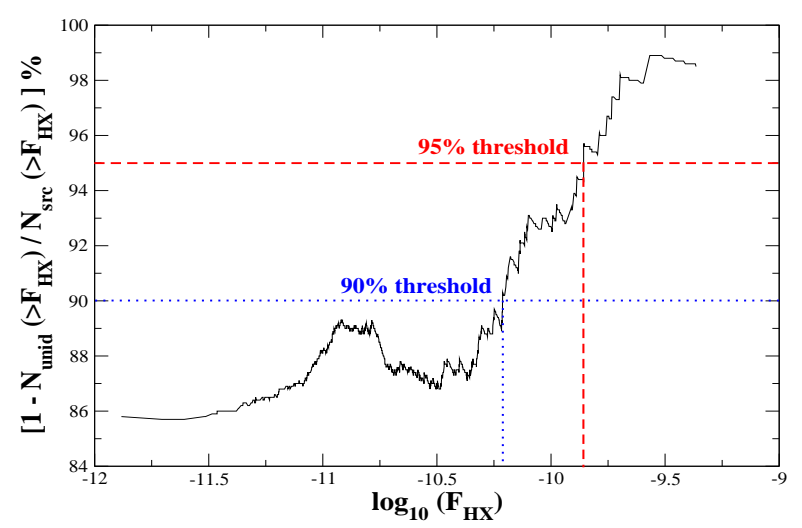

Fig. 1. Identification fraction of sources listed in the $3 \mathrm{PBC}$ computed as the ratio of the number of unidentified sources $\left(N_{\text {unid }}\right)$ to the total number of sources $\left(N_{\text {src }}\right)$ with flux in the hard X-ray band $15-150 \mathrm{keV}$, above a certain threshold. The blue and red lines indicate the hard X-ray flux for which the 3PBC has 90 and 95\% identification, respectively. About $85 \%$ of the sources in the $3 \mathrm{PBC}$ appear to have an optical spectroscopic classification.

terparts were associated by cross-matching their positions within $15^{\prime}$ of the soft X-ray sources listed in several catalogs.

We augmented both hard X-ray surveys (i.e., 3PBC and BAT105) by including hard X-ray unidentified sources classified as Seyfert galaxies during the optical spectroscopic campaign targeting low-energy counterparts of both Swift-BAT and INTEGRAL unidentified sources (see, e.g., Masetti et al. 2004, 2006, $2010,2012,2013$ ). Thanks to this campaign, we were able to find 44 additional Seyfert galaxies. This sample is less homogeneous than the previous ones, but follow-up spectroscopic observations complement the selection based on the 3PBC and the BAT105, thus motivating our choice of including them.

It is worth noting that $90 \%$ and $95 \%$ of the 3PBC sources have been classified and spectroscopically identified above 4.9 and $13 \times 10^{-11} \mathrm{erg} \mathrm{s}^{-1} \mathrm{~cm}^{-2}$ flux levels, respectively. In Fig. 1 we report the fraction of unidentified hard $\mathrm{X}$-ray sources over the total number of 3PBC sources above a certain hard X-ray flux threshold. The entire 3PBC appears to be classified at the level of $\sim 85 \%$, even if not all sources are spectroscopically identified and no multifrequency criteria were adopted.

\section{Selection criteria}

Given our primary goal of creating a catalog of Seyfert galaxies, with homogeneous selection criteria based on multifrequency observations, to investigate their properties having a negligible fraction of contaminants, we adopted the following minimum requirements for a source to be included in the Turin-SyCAT.

\subsection{Step-by-step procedure}

We inspected all sources belonging to surveys and catalogs previously listed (see Sect. 2), and we included only those selected according to the following criteria. Each step of the selection process is also summarized in the flow chart available in Fig. 2.

1. A published optical spectrum. This is to guarantee its classification, at least distinguishing between type 1 and type 2 Seyfert galaxies, and a definite redshift estimate. The 
Table 2. Number of sources in the starting catalogs or samples for the initial Seyfert galaxy selection.

\begin{tabular}{lrrrr}
\hline \hline Catalog & $\begin{array}{r}\text { Total } \\
\text { sources }\end{array}$ & $\begin{array}{r}\text { Seyfert } \\
\text { galaxies }\end{array}$ & $\begin{array}{r}\text { Targets } \\
\text { identified }\end{array}$ & \\
\hline 3PBC & 1593 & 520 & 265 & 300 \\
BAT105 & 1632 & 827 & 289 & 331 \\
\hline
\end{tabular}

reference for the spectrum inspected to check both redshift and classification is reported in Appendix A.

2. Radio luminosity lower than $10^{40} \mathrm{erg} \mathrm{s}^{-1}$ whenever there is a radio counterpart belonging to one of the major radio surveys, namely NVSS and SUMSS. We adopted this conservative criterion to exclude jetted AGNs (i.e., radio galaxies and blazars Capetti et al. 2017a,b; Heckman \& Best 2014).

3. A mid-IR counterpart listed in the AllWISE Source catalog. This allows us to have a uniform set of coordinates, flux, and magnitude measurements.

4. A $L_{3.4 \mu \mathrm{m}}<3 \times 10^{11} L_{\odot}$, as an arbitrary limit to differentiate Seyfert galaxies from quasars. This threshold is of the same order of magnitude as used in optical bands (Boroson \& Green 1992). Below this threshold there is an overlap in the luminosity distribution between quasars and Seyfert galaxies, although the former can reach luminosities above $10^{11} L_{\odot}$; thus, cutting our sample just using the infrared $L_{3.4 \mu \mathrm{m}}$ value guarantees avoiding bright quasars as contaminants of our selection ${ }^{4}$. We performed K-correction to the luminosity by assuming a spectral shape of a power law with spectral index calculated using magnitudes in the W1 and W2 WISE bands 5 .

The angular separation adopted to search for a radio counterpart in the major surveys are $11^{\prime \prime}$ and $8^{\prime \prime}$ for the NVSS and SUMSS, respectively. Previous analyses indicate that the probability of spurious associations at these angular separations is almost negligible (see, e.g., D'Abrusco et al. 2012, 2019). The lack of a radio counterpart in one of these surveys automatically implies that the source has a radio luminosity below our threshold of $10^{40} \mathrm{erg} \mathrm{s}^{-1}$ at redshifts lower than $\sim 0.4$, the same redshift range as the Turin-SyCAT.

Then, we cross-matched between WISE coordinates and the position in the original catalog or survey. We chose an angular separation of $3 \prime .3$, statistically derived and successfully adopted in our previous analyses (Massaro et al. 2012a, 2015b; D'Abrusco et al. 2013); this corresponds to a chance of spurious associations lower than 1\% (Massaro et al. 2011, 2013a). These cross-matches allowed us to assign all Seyfert galaxies the coordinates of their mid-IR counterparts so that their positions can be uniformly estimated. Hereafter we use WISE coordinates for all other cross-matches.

All numbers related to the initial sample of Seyfert galaxies extracted from each starting survey or catalog are summarized in Table 2 and are described as follows. Here we report the total number of sources in each catalog together with those classified as Seyfert galaxies, those selected as investigated, and the final number of sources identified and listed in the Turin-SyCAT.

\footnotetext{
4 It is worth noting that, for sources with both WISE and SDSS counterparts, the selection based on mid-IR and optical luminosities yields the same results.

5 https://ned.ipac.caltech.edu/level5/SeptQ2/Hogg/ frames.html
}

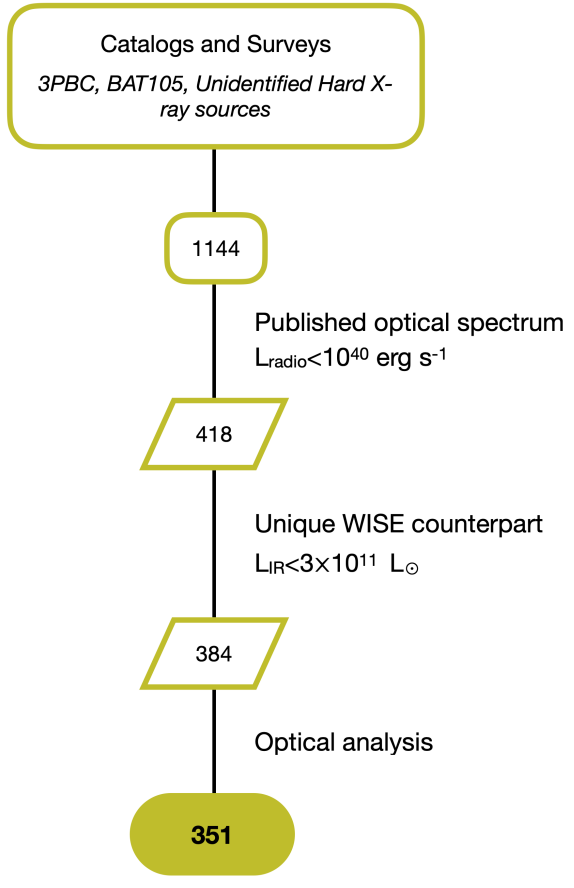

Fig. 2. Flow chart highlighting all steps and thresholds applied to select the final sample of Seyfert galaxies belonging to the first release of the Turin-SyCAT. We selected sources with radio luminosity lower than $10^{40} \mathrm{erg} \mathrm{s}^{-1}$ to avoid jetted AGNs and $L_{3.4 \mu \mathrm{m}}<10^{11} L_{\odot}$ to avoid quasars.

- The 3PBC catalog lists a total of 1593 hard X-ray sources with 534 unidentified sources, 356 of which are associated with a low-energy counterpart classified as Seyfert 1 and 164 as Seyfert 2.When inspecting this catalog we only extracted a total of 265 Seyfert galaxies that met our criteria. An additional 35 sources were also identified thanks to the optical spectroscopic observations carried out for the unidentified hard X-ray sources (see, e.g., Masetti et al. 2013, and references therein).

- The BAT105 catalog lists 827 sources classified as Seyfert galaxies. Here we selected a total of 289 Seyfert galaxies plus an additional 42 classified as Seyfert galaxies from those observed during the optical spectroscopic campaign of unidentified hard X-ray sources. In particular, 280 Seyfert galaxies belong to both the 3PBC and the BAT105 catalog.

The total number of Seyfert galaxies selected from the previous surveys and catalog was 351.

We note that for each sample listed above the number of selected Seyfert galaxies is significantly lower than the original one. The main reasons underlying this discrepancy are mainly due to the fact that we classify as Seyfert galaxies only those sources with radio power lower than $10^{40} \mathrm{erg} \mathrm{s}^{-1}$ and that we found a significant number of sources lacking an optical spectrum such that we could not verify the $z$ estimate and its proper classification.

We note that the Seyfert classification adopted, for example, in the Swift-BAT catalogs 3PBC and BAT105, is often misleading since it is based only on the optical properties of the hard X-ray counterparts; there are many radio galaxies belonging to the Third Cambridge Catalog (3C; Edge et al. 1959) or the Fourth Cambridge catalog (4C; Gower et al. 1967; Pilkington \& Scott 1965) erroneously classified as Seyfert galaxies but whose whole multifrequency behavior is certainly more similar to radio galaxies. 

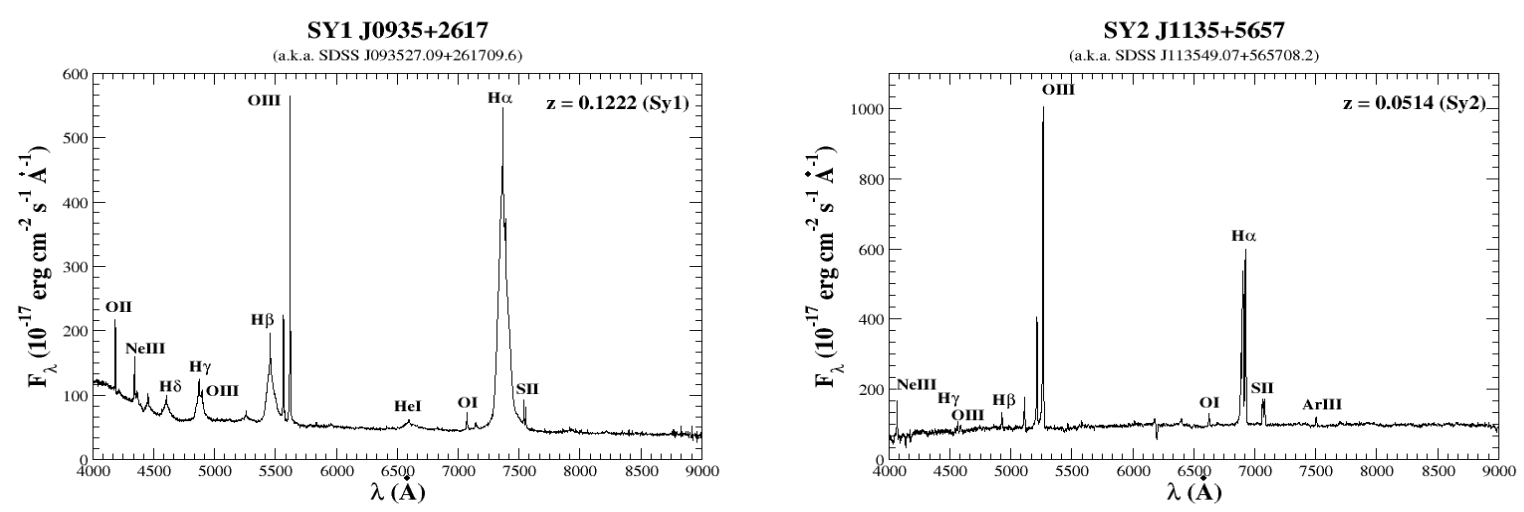

Fig. 3. Example of optical spectra of type 1 and type 2 Seyfert galaxies. Left panel: optical spectrum of a classical type 1 Seyfert galaxy: SY1 J0935+2617 (also known as SDSS J093527.09+261709.6) listed in the Turin-SyCAT where broad emission lines are clearly seen in the range 4000-9000 Å as indicated. Right panel: same as left panel, but for a typical type 2 Seyfert galaxy: SY2 J1135+5657 (SDSS J113549.08+565708.2), lacking broad emission lines. The main spectral emission and/or absorption features are labeled in both figures, thus providing clear examples of those spectra searched for in the literature to prepare the Turin-SyCAT.

We reclassified the sources classified as quasars according to our multifrequency criteria. The proportion of quasars found in the input catalogs was less than $1 \%$, given the luminosity cut adopted at 3.4 microns. Based on the multifrequency information we discarded those radio galaxies and quasars.

\subsection{Optical spectroscopic classification}

Our optical spectroscopic classification, type 1 or 2 , is based on the criteria outlined by Khachikian \& Weedman (1974). In Fig. 3 we show two typical examples to highlight the main optical emission lines present. For the Turin-SyCAT we selected only those sources for which at least three co-authors agree on the same Seyfert galaxy classification inspecting their optical spectra.

Both classes of Seyfert galaxies show the presence of narrow emission lines (widths of $\sim 10^{2} \mathrm{~km} \mathrm{~s}^{-1}$ ) in their optical spectra interpreted as being due to low-density $\left(n_{\mathrm{e}} \sim 10^{3}-10^{6} \mathrm{~cm}^{-3}\right)$ gas clouds photoionized by the central source (Koski 1978; Ferland \& Netzer 1983; Stasińska 1984). Broad emission lines (widths up to $10^{4} \mathrm{~km} \mathrm{~s}^{-1}$ ), due to permitted transition, are visible only in type 1 Seyfert galaxies where the absence of broad forbidden emission lines indicates relatively high-density (i.e., $n_{\mathrm{e}}>10^{9} \mathrm{~cm}^{-3}$ ) gas clouds.

In addition weak absorption features due to late-type giant stars in the host galaxy are also observed in both type 1 and type 2 Seyfert spectra; absorption lines are relatively weak because the starlight is diluted by the non-stellar featureless continuum. The AGN continuum is usually so weak in Seyfert 2 galaxies that it is quite difficult to isolate it from the stellar continuum unambiguously (Storchi-Bergmann et al. 1998; Contini \& Viegas 2000).

The final number of Seyfert galaxies listed in this first release of the Turin-SyCAT is 351: 233 type 1 and 118 type 2 . In the current release, we only distinguished between type 1 and type 2 Seyfert galaxies. Intermediate types (i.e., as subclasses of Seyfert $1.2,1.5,1.8$, and 1.9 ) for which there is a detection of a broad component were all classified as type 1 . We do not provide the intermediate classification in the current release since we do not have homogeneous spectral data for all to refine our classification. However, we will present a refinement of the spectral classification at least for those Seyfert galaxies lying in the SDSS footprint in future releases of the Turin-SyCAT.

It is also worth noting that narrow-line spectra are clearly distinguishable from the HII-region spectra seen in normal galaxies.
Seyfert galaxies show a wide range in ionization level, which is typical of a gas ionized by a source where the input continuum spectrum falls off slowly (relative to a Wien law) at ionizing wavelengths. On the other hand, on the basis of the data collected to date, it was not possible to distinguish Seyfert galaxies from low-luminosity active galaxies classified as LINERs (Baldwin et al. 1981; Kauffmann et al. 2003; Kewley et al. 2006).

\section{The Turin-SyCAT}

We labeled all sources listed in the first release of the TurinSyCAT with a prefix Sy1 or Sy2 following by coordinates in the format $\mathrm{J} 0000+0000$ to have a unique identifying name. For only 10 sources out of 351, our optical classification was different from the one reported in the literature; in any case, both classifications are reported in the final catalog table available in Appendix A. We also note that narrow-line Seyfert 1 galaxies (Osterbrock \& Pogge 1985) are labeled in our catalog as Sy1.

In Fig. 4 the lack of type 2 Seyfert galaxies above $z=0.1$ is quite evident, and that at $z>0.04$ the fraction of type 2 to type 1 Seyfert drastically drops, suggesting that the samples and catalogs used to create the Turin-SyCAT could be incomplete.

The sky distribution, computed using the Aitoff projection, of all 351 Turin-SyCAT sources is then reported in Fig. 5 where a larger fraction of sources (203) have declinations $\delta>0^{\circ}$, where it is to easier to get access to optical telescopes to perform source identifications (Green et al. 1986; Colless et al. 2001; Jones et al. 2004; Aguado et al. 2019).

All Seyfert galaxies are detected in all four WISE bands. In the near-IR (i.e., 2MASS, automatically associated in the AllWISE catalog), we have 350 sources detected in $J$ band and $H$ band, and 347 in Ks band out of a total of 351, with only 1 source (SY2 J0952-6232) lacking a 2MASS counterpart.

The number of Seyfert galaxies within the SDSS footprint is 193 out of 351; all of them having a unique cross-match within $1^{\prime \prime}$. Three associations at larger angular separations are SY2 $\mathrm{J} 1001+5540$, SY1 J1136+2135, and SY2 J1225+1239, having the SDSS counterpart at 4".241, 2".88, and 1'.51 from their WISE positions, respectively. In the SDSS footprint are 78 out of 193 Seyfert galaxies with redshift estimates. For all of them we compared SDSS redshift values with those obtained in the literature and inspected by us during the selection process. The redshift 


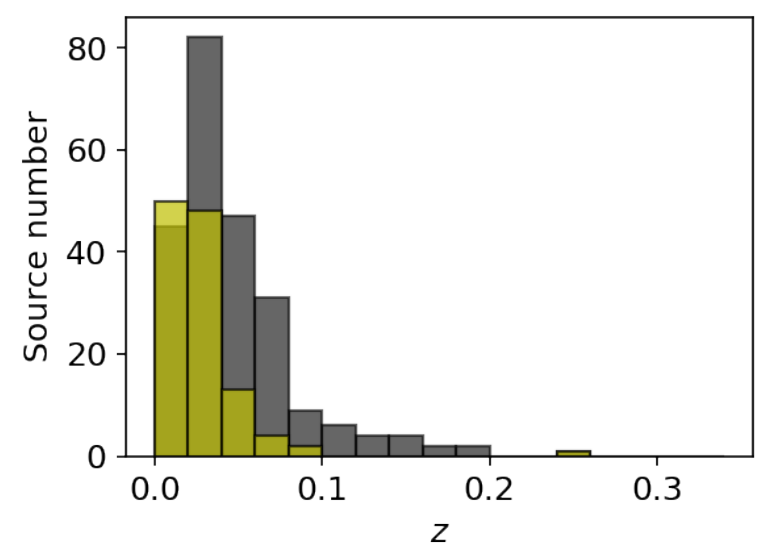

Fig. 4. Redshift distribution of all Seyfert galaxies included in the first release of the Turin-SyCAT. Seyfert galaxies classified as type 1 are shown in black, while those classified as type 2 are in yellow. (This color scheme is adopted through the entire manuscript). It is worth noting that type 2 Seyferts are basically not selected at $z>0.1$.

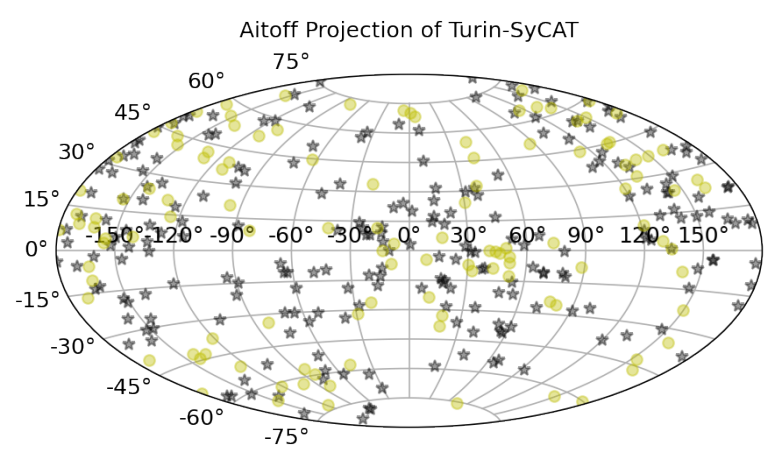

Fig. 5. Sky distribution of all 351 Seyfert galaxies listed in the TurinSyCAT, in the Aitoff projection. Seyfert 1 are represented as black stars and Seyfert 2 as yellow circles.

differences between those reported in the SDSS and those we collected are all less than $10^{-3}$.

Once we obtain uniform coordinates for all sources in TurinSyCAT, thanks to their WISE counterparts, we cross-matched our catalog with the Fourth IBIS/ISGRI Soft Gamma-Ray Survey Catalog 6 (IBISCAT4; Bird et al. 2007), and with the ROSAT Bright and Faint Source catalogs (RBSC and FRSC, respectively; Voges et al. 1999, 2000). To carry out all these crossmatches, we adopted a conservative positional uncertainty for WISE coordinates of 1 ", much larger than that reported in the AllWISE catalog (Wright et al. 2010), but our results are not affected significantly by this choice. All matches are also unique, as expected by the low sky density of the hard X-ray sources.

In the hard X-ray sky, we found that 102 out of 351 Seyfert galaxies lie within the positional uncertainty of IBISCAT4 sources; this result was found using the positional uncertainty reported in the IBISCAT4. Finally, from the cross-match with the RBSC and the FRSC catalogs (Voges et al. 1999, 2000) finding that 160 Seyfert galaxies out of 351 have a soft X-ray counterpart, a unique match, within the positional uncertainty reported for the X-ray coordinates and 1 " for AllWISE coordinates, as previously considered.

Finally, Fig. 6 shows the Venn diagram of the Seyfert galaxies selected for the first release of the Turin-SyCAT having a

\footnotetext{
6 https://heasarc.gsfc.nasa.gov/W3Browse/integral/ ibiscat $4 . h$ tml
}

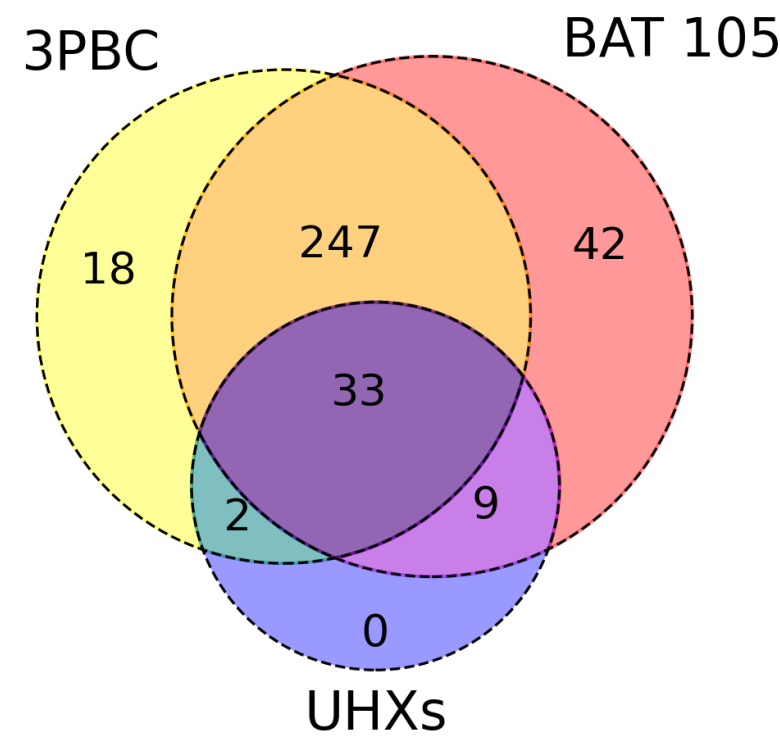

Fig. 6. Venn diagram of sources selected for Turin-SyCAT and associated in both the 3PBC and the BAT105, highlighting those discovered thanks to the optical spectroscopic campaign of unidentified hard X-ray sources, is shown.

hard X-ray counterpart in the 3PBC and/or in the BAT105 catalogs or discovered thanks to the optical spectroscopic campaign of unidentified hard X-ray sources.

\section{Characterizing the Seyfert galaxy population}

\subsection{Infrared emission}

We compared the mid-IR colors of type 1 and type 2 Seyfert galaxies listed in this first release of the Turin-SyCAT (see Fig. 7) since all selected sources have a WISE counterpart detected in all four WISE bands with only one exception (see Sect. 3 for additional details).

The distribution of the W1-W2 color appears to be more concentrated for the Seyfert galaxies of type 1, while that of type 2 covers a broader range. No clear differences are visible in both WISE color diagrams, with the only exception that overall Seyfert 2 galaxies tend to be redder than Seyfert 1. More than $90 \%$ of type 1 galaxies and $\sim 50 \%$ of type 2 have the midIR color $W 1-W 2>0.5$, the same criterion used to identify and select AGNs from other sources (Ashby et al. 2009), while adopting a more conservative threshold (low contamination from other source classes) $\sim 75 \%$ of Seyfert 1 galaxies and $\sim 40 \%$ of Seyfert 2 show $W 1-W 2>0.8$ (Stern et al. 2012).

To test that such differences are not related to the redshift distributions of type 1 and type 2 Seyfert galaxies we also compared their IR colors in a restricted redshift range of 0.01 bin size, and we confirmed the previous results (see Appendix B for more details).

Then, given the recent and extensive use of these midIR color-color diagrams to select $\gamma$-ray blazar-like candidates that could be potential counterparts of unidentified $\gamma$-ray sources (Massaro et al. 2012a, 2013a; D’Abrusco et al. 2013; Massaro \& D'Abrusco 2016) and targets of optical campaigns (Paggi et al. 2014; Massaro et al. 2014, 2015c; Landoni et al. 2015; Álvarez Crespo et al. 2016; Marchesini et al. 2016, 2019a; Paiano et al. 2017, 2019; Marchesi et al. 2018; Peña-Herazo et al. 2017, 2019), we also compared the mid-IR colors of Seyfert galaxies with those of both BL Lac objects and flat spectrum radio 

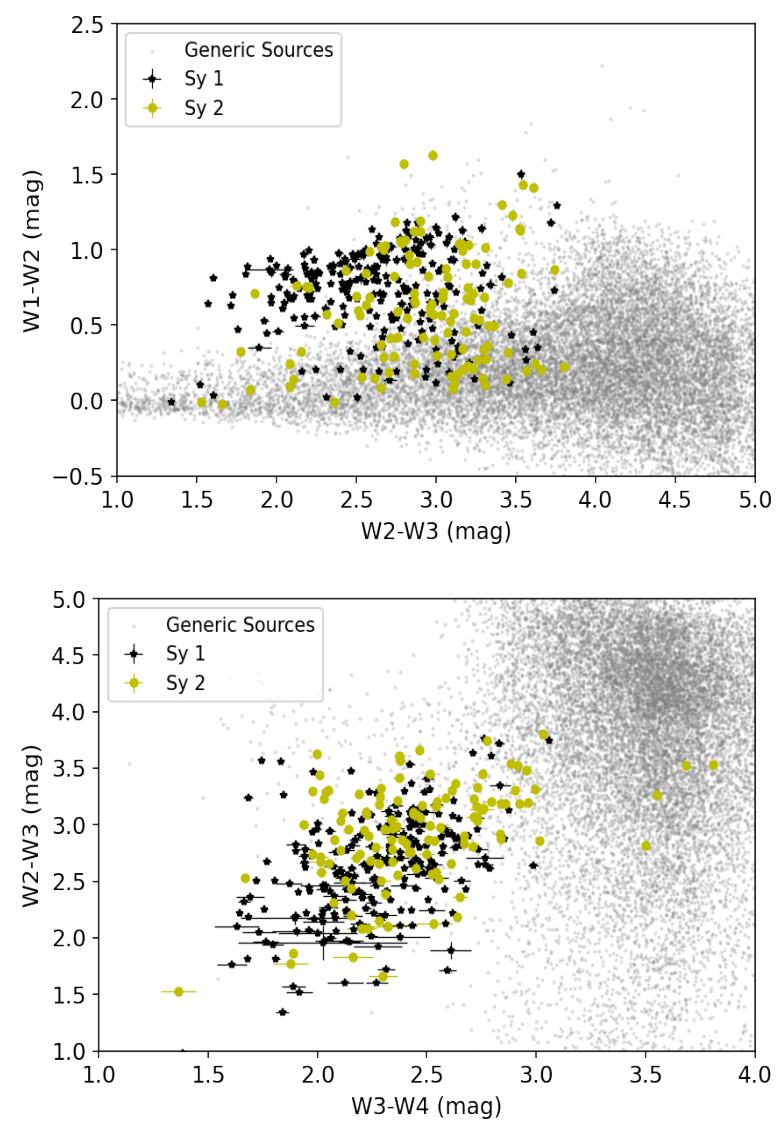

Fig. 7. Color-color diagrams based on the WISE magnitudes comparing Seyfert galaxies of type 1 (black) and type 2 (yellow) with generic midIR sources selected in a random region of the sky (see Massaro et al. 2011, 2013a,b, 2016, for a similar analysis). Seyfert galaxies of type 2 appear to be redder than those of type 1 , and are quite separated in the W1-W2 vs. W2-W3 color-color plot in the left lower.

quasars, labeled BZBs and BZQs, respectively, listed in the latest release of Roma-BZCAT (Massaro et al. 2009, 2015a) and associated with a Fermi source in the Fourth Fermi-LAT Source catalog (Abdollahi et al.2020). This comparison is shown in Fig. 8, where it is clear that in the W1-W2 versus W2-W3 diagrams Seyfert 1 galaxies appear to lie in the middle between BZBs and BZQs, a fraction of them with similar colors, while type 2 Seyfert galaxies have mid-IR colors very distinct from those of blazars. On the other hand, in the W2-W3 versus W3-W4 plane, Seyfert galaxies of type 1 are more similar to BZQs, and the same situation occurs for type 2 Seyfert galaxies. It is worth noting that even if there is a fraction of Seyfert galaxies showing mid-IR colors similar to known $\gamma$-ray blazars, as previously noted in de Menezes et al. (2019), these sources do not contaminate the selection of $\gamma$-ray blazar candidates significantly since this is also based on the radioloudness parameter (see D'Abrusco et al. 2014, for more details).

For Turin-SyCAT sources having a counterpart in 2MASS, we also show their near-IR properties in Fig. 9. As in the previous color-color diagrams, Seyfert 2 galaxies are redder than type 1 sources, but even if they are very distinct from background sources, they show quite similar properties.

\subsection{Optical properties}

Given the significant number of Seyfert galaxies lying in the SDSS footprint selected for the first release of the Turin-SyCAT (see Sect. 3), we also compared their optical colors with their mid- and near-IR colors, as shown in Fig. 10.

Seyfert galaxies are distinct in their $u-r$ color distribution, as expected given their spectral properties, with Seyfert 1 galaxies being bluer than type 2 sources.

Since all sources listed in the Turin-SyCAT at declinations above $-33^{\circ}$ also lie in the Pan-STARRS footprint, for a total of 294 counterparts within $1^{\prime \prime} .5$, we also inspected this information, but we did not find any additional insight with respect to those already obtainable from SDSS. However, consistently with the mid-IR luminosities, all the $R$-band optical luminosities computed using the Pan-STARRS measurements lie in the range between $10^{8}$ and $10^{11} L_{\odot}$ (K corrected). It is worth noting that for all Seyfert galaxies lying both in the Pan-STARRS and SDSS footprints, their $R$-band magnitudes are consistent within $3 \sigma$ uncertainty. We also note that for all Seyfert galaxies with a SDSS counterpart the optical luminosity in the $R$ band is lower than $10^{11} L_{\odot}$. Thus, we can confirm that we did not include bright quasars.

\subsection{The IR - hard X-ray connection}

Restricting the sample to those Seyfert galaxies with a hard $\mathrm{X}$-ray counterpart on the $3 \mathrm{PBC}$, we also performed an analysis comparing mid-IR properties with those in the $15-150 \mathrm{keV}$ energy range. First, we computed the distribution of ratios between their flux at $12 \mu \mathrm{m}$ and $22 \mu \mathrm{m}$ (i.e., $F_{12}$ and $F_{22}$ ), and that measured in the hard X-rays (i.e., $F_{H X}$ ) between $15 \mathrm{keV}$ and $150 \mathrm{keV}$, for which we adopted the measurements reported in the 3PBC (see Fig. 11). Our choice of using the 3PBC rather than the BAT105 catalog was motivated by the fact that it has a higher identification fraction given its higher flux limit.

Investigating the connection between the hard X-ray and the mid-IR emission in Seyfert galaxies is crucial since highenergy emission measures an intrinsic radiated luminosity above $\sim 10 \mathrm{keV}$, while WISE $12 \mu \mathrm{m}$ and $22 \mu \mathrm{m}$ is related to the reprocessed radiation from the dust of all energy absorbed from the optical and UV wavelengths. We did not find any significant difference between the distributions of the infrared-to-hard X-ray flux ratio in Seyfert 1 and Seyfert 2 galaxies according to the Kolmogorov-Smirnov test $(p$-value $=0.09)$.

On the other hand, we found two significant trends when comparing the mid-IR fluxes with those in the hard X-ray bands, as shown in Fig. 12. The correlation between $F_{12}$ and $F_{H X}$ has a linear correlation coefficient of 0.57 (slope of $1.02 \pm 0.10$ ) for Seyfert 1 galaxies and 0.52 (slope of $0.93 \pm 0.16$ ) for Seyfert 2. In both cases, there is a negligible probability of being spurious.

Similar values for the correlation between $F_{22}$ and $F_{H X}$ being 0.59 (with a slope of $1.05 \pm 0.10$ ) for type 1 Seyfert galaxies and 0.49 (slope of $0.95 \pm 0.17$ ) for type 2 Seyfert galaxies, with again a probability of less than $10^{-6}$. Considering both classes together, given their similar mid-IR to hard X-ray ratios, we found a correlation coefficient of 0.57 and 0.55 (slopes of $0.93 \pm 0.08$ and $0.95 \pm 0.08$ ) for the hard X-ray flux $F_{H X}$ correlating with $F_{12}$ and $F_{22}$, respectively. We also show, in blue, the correlation for the $90 \%$ complete subsample with slopes of $0.94 \pm 0.41$ (linear correlation coefficient of 0.40 ) and $1.14 \pm 0.49$ (linear correlation coefficient of 0.41 ) for $F_{12}$ and $F_{22}$, respectively. When considering type 1 and 2 together, the slopes are $1.01 \pm 0.09$ (linear correlation coefficient of 0.57 ) and $1.04 \pm 0.09$ (linear correlation coefficient of 0.55 ) for $F_{12}$ and $F_{22}$, respectively.

Our results are consistent with other literature analyses that found a correlation between the mid-IR and the hard X-ray 

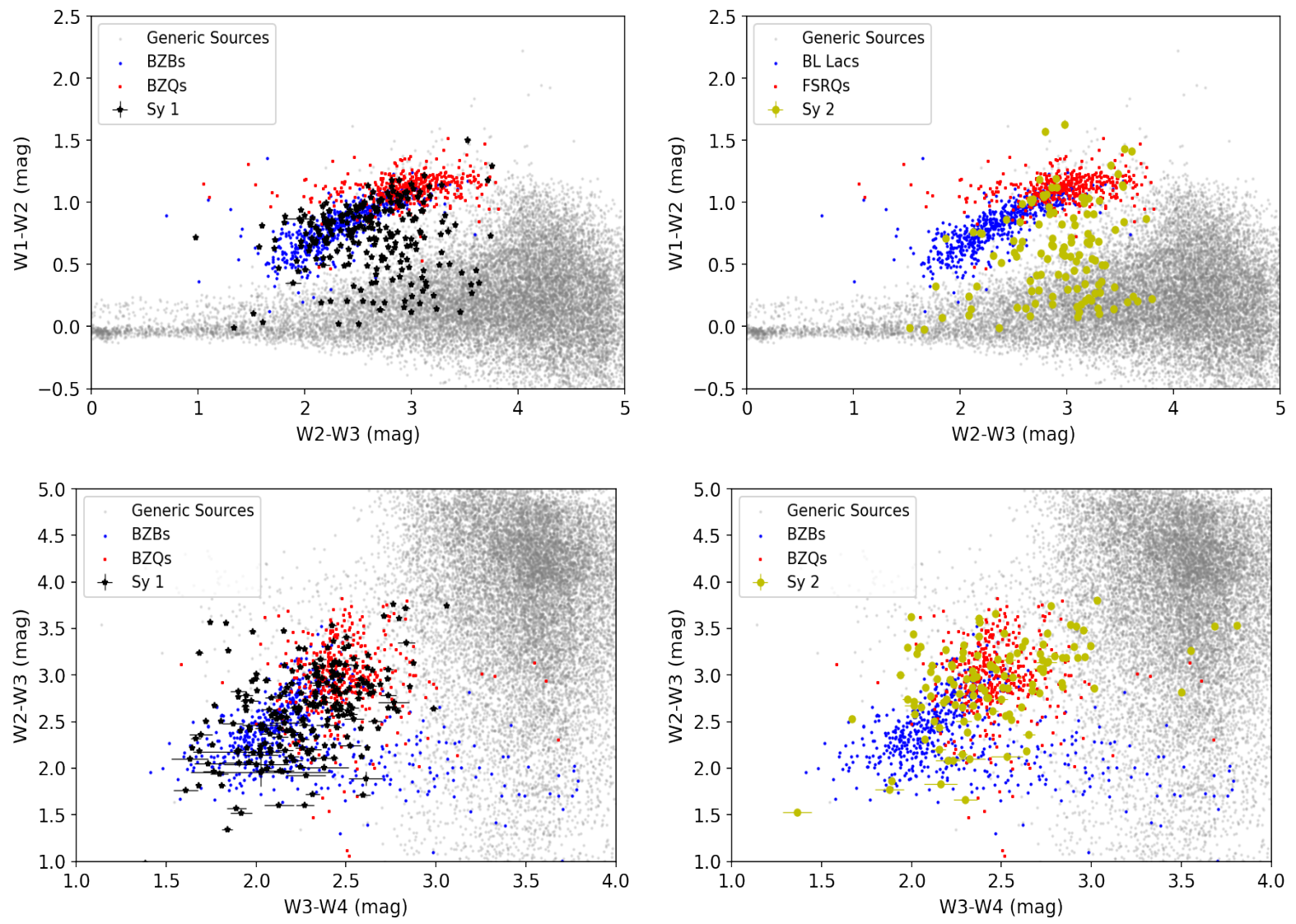

Fig. 8. Comparison between the mid-IR colors of Seyfert galaxies listed in the Turin-SyCAT and the $\gamma$-ray blazars of the Roma-BZCAT. Seyfert 1 galaxies tend to be more similar to blazars in their mid-IR emission, in particular when compared with BZQs that have the same infrared and optical properties of normal quasars, while Seyfert 2 galaxies show mid-IR properties similar to BZQs only in the W2-W3 vs. W3-W4 diagram.

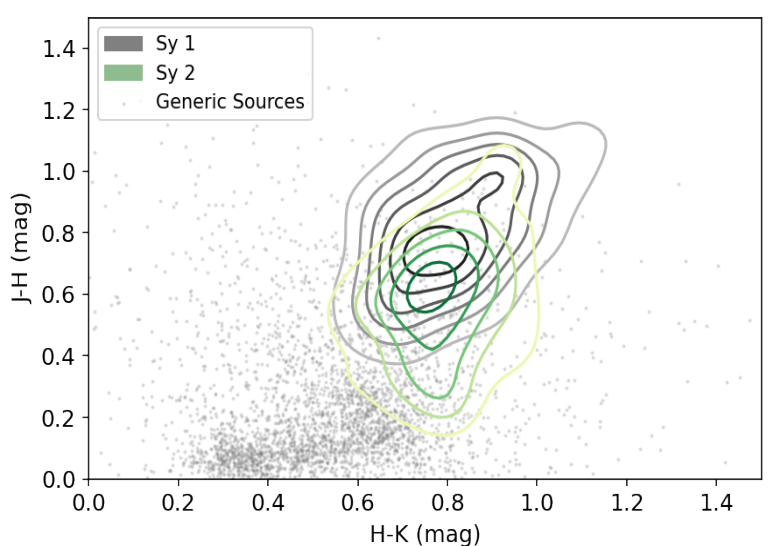

Fig. 9. Color-color diagrams based on the 2MASS magnitudes comparing contours of Seyfert galaxies of type 1 (gray-to-black) and type 2 (yellow-to-green) with generic sources selected in a random region of the sky (see Massaro et al. 2011, for a similar analysis). While Seyfert galaxies occupy a distinct region from that of generic infrared sources their near-IR properties are quite similar. Isodensity contours are computed using KDE (see, e.g., D’Abrusco et al. 2019).

luminosity in AGNs (Ichikawa et al. 2017, 2019), where the sample was selected from the Swift-BAT 70-month catalog (Baumgartner et al. 2013). Our results are also consistent with those of Gandhi et al. (2009) and Asmus et al. (2015); however, literature analyses are carried out over all AGNs emitting in the hard X-rays here, while for the first time we restricted our analy- sis only to Seyfert galaxies, and spanning a narrow redshift range but focusing on a precise population.

This correlation was also found for AGNs with luminosities in the hard X-ray band $14-195 \mathrm{keV}, L_{14-195}<10^{44} \mathrm{erg} \mathrm{s}^{-1}$ with data collected from several X-ray observatories (ASCA, BeppoSAX, Chandra, and XMM-Newton) and compared with Infrared Space Observatory data (samples sizes of 57 and 71, respectively; Lutz et al. 2004; Ramos Almeida et al. 2007) or using Spitzer compared with INTEGRAL (sample size of 68 AGNs, Sazonov et al. 2012).

Carrying out this analysis, we also compared the hard X-ray photon index $\Gamma_{H X}$ with all mid-IR colors, but we did not find any significant trend and the distribution of $\Gamma_{H X}$ appear to be the same when comparing type 1 and type 2 Seyfert galaxies.

On the other hand, we also found a correlation between the mid-IR flux computed at $3.4 \mu \mathrm{m}$ and $F_{H X}$ (correlation coefficient equal to 0.67 ) as for other mid-IR fluxes, but only a mild trend (correlation coefficient equal to 0.45 ) is present when comparing near-IR fluxes computed from 2MASS magnitudes and $F_{H X}$, both with negligible $\mathrm{p}$-chance values. We note that the underlying reason to choose to show only results related to the $3 \mathrm{PBC}$ catalog with respect to BAT105 and IBISCAT4 samples is that the former does not have positional uncertainty reported.

As an additional test we also cross-matched our SYCAT sources with the Point Source catalog of the Infrared Astronomical Satellite (IRAS) ${ }^{7}$ using all the elliptical positional

\footnotetext{
7 https://heasarc.gsfc.nasa.gov/W3Browse/iras/iraspsc.
} html 

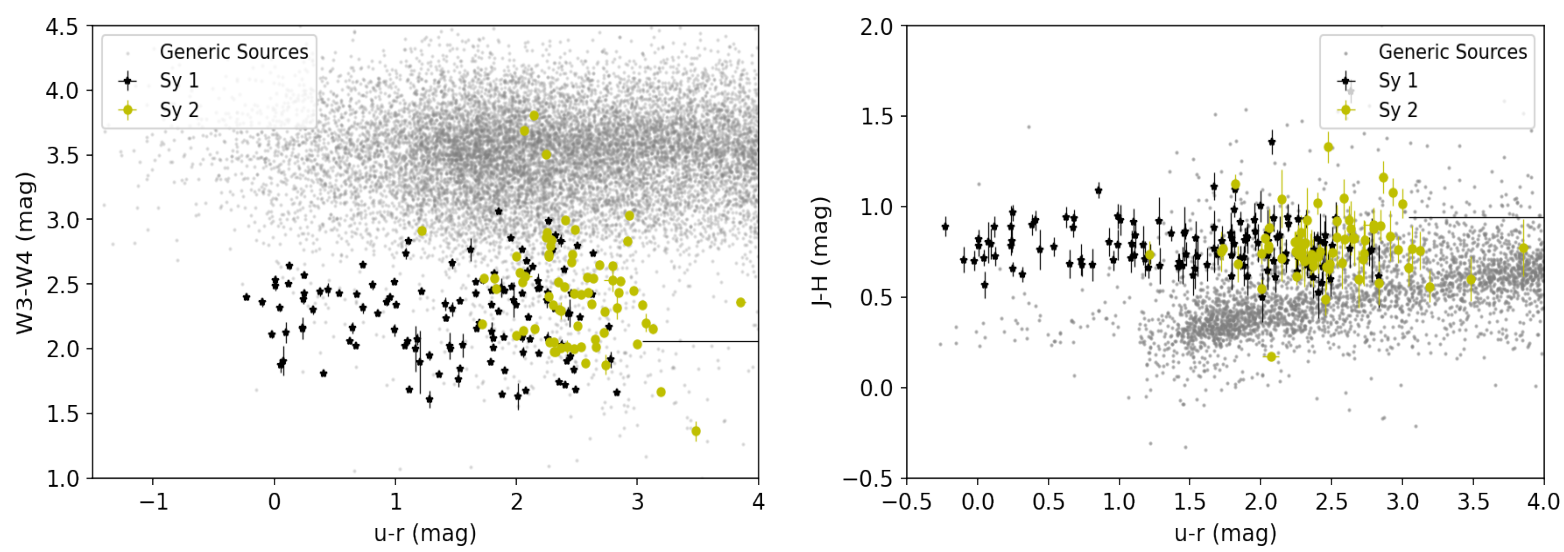

Fig. 10. Comparison between the optical and the mid-IR (left panel) and near-IR (right panel) colors for both Seyfert galaxies of type 1 (black in both plots) and type 2 (yellow in both plots). Seyfert galaxies are clearly separated in two distinct regions of both plots when considering the $u-r$ color. These color-color diagrams are drawn only for those Seyfert galaxies lying in the SDSS footprint (see Sect. 3).
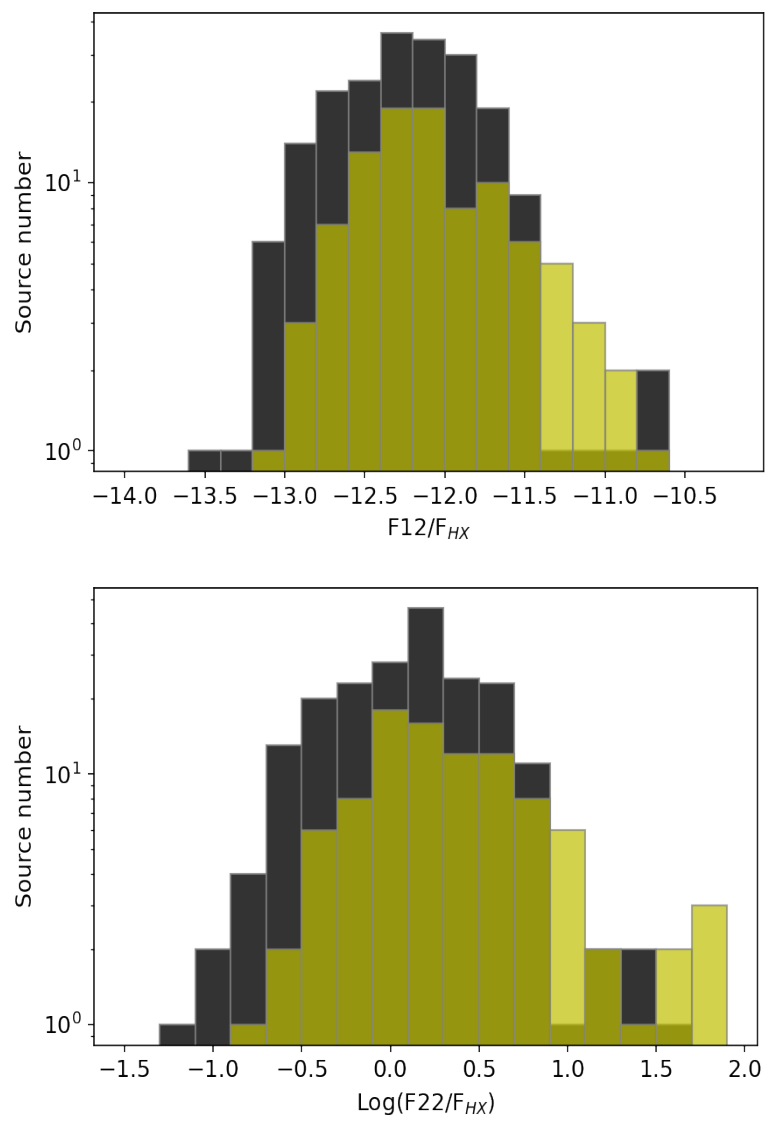

Fig. 11. Distributions of the ratios between the integrated flux in the WISE band centered at the nominal wavelength of $12 \mu \mathrm{m}$ (top panel) and $22 \mu \mathrm{m}$ (bottom panel), over the integrated flux in the hard X-ray band $F_{H X}$ between 15 and $150 \mathrm{keV}$ collected for all Seyfert galaxies with a counterpart in the 3PBC and selected to belong to the TurinSyCAT. There is no clear difference between the distributions of type 1 and type 2 Seyfert galaxies appearing in black and yellow, respectively.

uncertainty regions listed there. We found that 72 Seyfert 1 and 77 Seyfert 2 galaxies have a unique IRAS counterpart at $60 \mu \mathrm{m}$ and $100 \mu \mathrm{m}$. Then we searched for a trend between the flux at $60 \mu \mathrm{m}$, and $100 \mu \mathrm{m}$ (i.e., $F_{60}$ and $F_{100}$, respectively) and that measured in hard X-rays from the $3 \mathrm{PBC}$, only for those Seyfert galaxies with a $3 \mathrm{PBC}$ counterpart, as previously performed for
WISE. As shown in Fig. 13, no clear trend or correlation was found. This is in agreement with our results since cold dust is mostly related to the star formation in SYCAT sources, and it is not expected to be linked directly with the central AGN.

\section{Summary, conclusions, and future perspectives}

The main goal of the study presented here is to create a cata$\log$ of Seyfert galaxies with homogeneous and stringent selection criteria based on multifrquency observations. This allows us to check source identification and classification for hard X-ray sources and to search for trends or correlations between different observed quantities.

Our initial selection is based on the two main hard X-ray surveys, namely the third release of the Palermo BAT source catalog (Cusumano et al. 2010) and the BAT 105-month survey (Oh et al. 2018) carried out again using the data collected by the Swift-BAT instrument. Both hard X-ray surveys are also augmented by introducing additional Seyfert galaxies discovered thanks to a follow-up spectroscopic campaign carried out on the unidentified hard X-ray sources, whose aim was to search for low-energy counterparts of unidentified sources (Masetti et al. 2004, 2006, 2009, 2010, 2012, 2013; Parisi et al. 2012; Rojas et al. 2017; Marchesini et al. 2019b).

Applying our selection criteria (see Sect. 4 for details), we built the first release of the Turin-SyCAT, listing 351 Seyfert galaxies: 233 type 1 and 118 type 2 . Then, thanks to all crossmatches performed with existing databases, we also carried out a multifrequency analysis of the Seyfert galaxy population. All the sources listed in the Turin-SyCAT have a published spectrum that allowed us to firmly establish their redshifts and their optical spectroscopic classifications, they all have an associated mid-IR counterpart in the AllWISE catalog, and they all have radio luminosity values, when associated with a radio counterpart in one of the major surveys (i.e., NVSS and SUMSS), of less than $10^{40} \mathrm{erg} \mathrm{s}^{-1}$ and mid-IR luminosity values computed at $3.4 \mu \mathrm{m}$ lower than $3 \times 10^{11} L_{\odot}$. References for their redshifts and for their spectra used to classify the source and for a literature comparison are all reported in Appendix A.

Results of the multifrequency characterization for all Seyfert galaxies listed in the Turin-SyCAT can be summarized as follows:

- We selected 351 Seyfert galaxies with homogeneous properties at radio, infrared, and optical energies. All Seyfert 

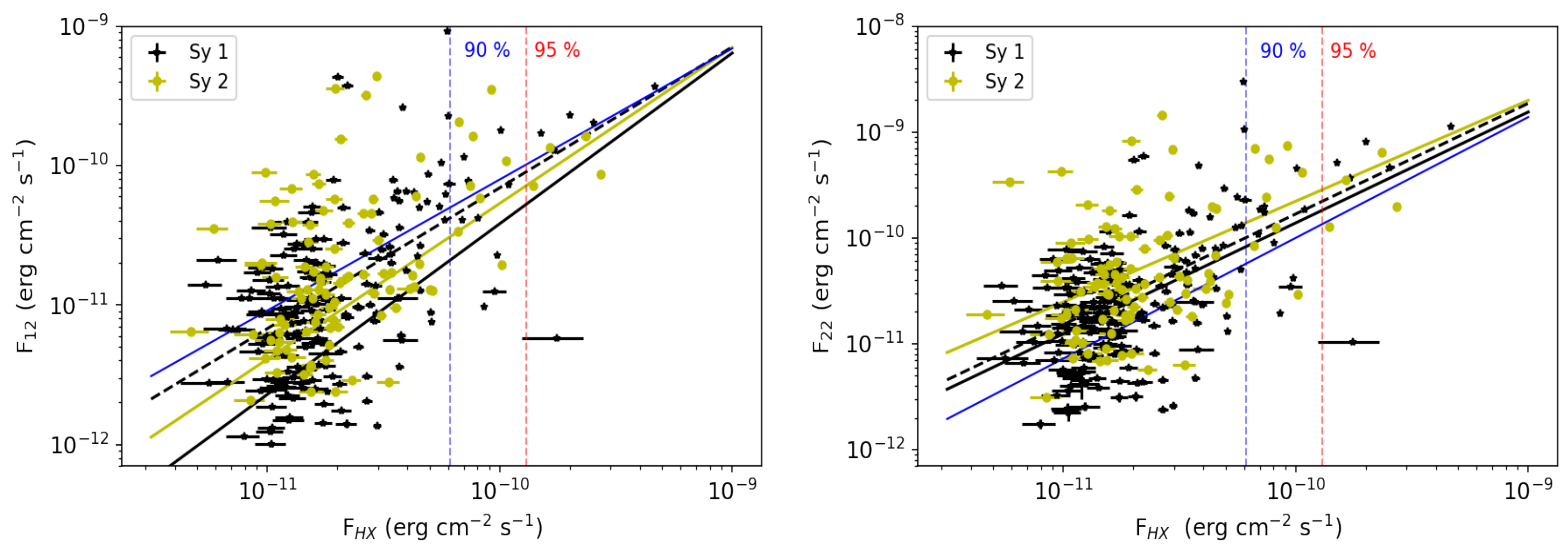

Fig. 12. Mid-IR fluxes at $12 \mu \mathrm{m}$ and $22 \mu \mathrm{m}$ as a function of hard X-rays flux. Left panel: Correlation found between the W3 integrated flux and that in the hard X-ray band from the 3PBC, for both Seyfert 1 and 2 galaxies (marked in black and yellow, respectively). The dashed black line corresponds to the regression line computed for the whole sample while the straight black and yellow lines mark that for type 1 and type 2 Seyfert galaxies, respectively. The correlation coefficients are reported in Sect. 5.3, while the p-chance values are negligible, all being lower than $10^{-6}$. Right panel: same as left plot, but computed using the mid-IR flux at $22 \mu \mathrm{m}$. The vertical dashed blue and red lines indicate the flux at which the sample is complete to $90 \%$ and $95 \%$, respectively.
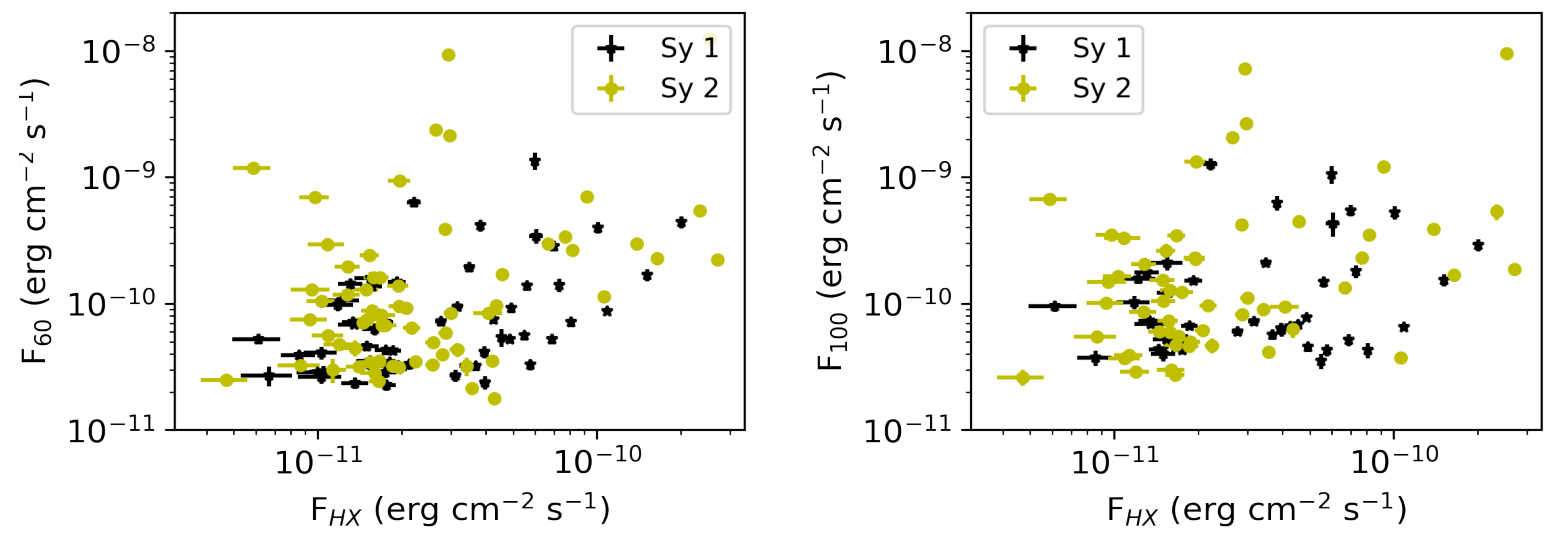

Fig. 13. Far-IR fluxes at $60 \mu \mathrm{m}$ and $100 \mu \mathrm{m}$ as a function of hard X-rays flux. Left panel: Scatter plot for the integrated flux at $60 \mu \mathrm{m}$ collected from the IRAS Point source catalog (i.e., $F_{60}$ ) and that in the hard X-ray band (i.e., $F_{H X}$ ), as listed in the 3PBC, for both Seyfert 1 and 2 galaxies (marked in black and yellow, respectively, as shown in previous figures). No clear trend or correlation is evident. Right panel: same as left plot, but computed using the far-IR flux at $100 \mu \mathrm{m}$ (i.e., $\left.F_{100}\right)$.

galaxies are classified as type 1 and type 2 , and all their redshifts were verified.

- Type 1 Seyfert galaxies could have mid-IR colors similar to blazars, but being radio quiet they are barely selected in cata$\log$ s of $\gamma$-ray blazar candidates. Seyfert 2 galaxies have midIR colors more similar to quasars than to BL Lac objects.

- For those Turin-SyCAT sources with a counterpart in the SDSS footprint, we also highlight that, in agreement with their spectral properties, type 1 and 2 Seyfert galaxies have a neat distinction when using the $u-r$ color.

We also tested that differences in the color-color diagrams are not due to differences in the redshift distribution between type 1 and type 2 Seyfert galaxies.

Comparing the mid-IR properties of those Seyfert galaxies having a $3 \mathrm{PBC}$ counterpart only, we found a tight correlation between the mid-IR flux (in the W3 and W4 bands) and that in the 15-150 keV energy range for both classes of Seyfert galaxies. The ratio of these two integrated fluxes is also remarkably similar for both Seyfert types. The correlation is also present when considering the mid-IR flux at $3.4 \mu \mathrm{m}$ but has less significance when using the near IR fluxes collected thanks to the 2MASS cross-matches. All these correlations are in agreement with expectations of the unification scenario of type 1 and type
2 Seyfert galaxies, where the fraction of luminosity absorbed by dusty components should be the same for both Seyfert types and the differences mainly related to the viewing direction.

In addition, we did not find any trend between far-IR fluxes and hard X-ray ones for all SyCAT sources with a counterpart in the IRAS Point Source Catalog (193 out of 517). In agreement with the fact that relatively cold dust emits at longer wavelengths than those observed by WISE, it is not directly related to the central AGN and the dusty torus obscuring it. We plan to carry out a further investigation of the mid-IR versus hard X-ray connection using the Combined Atlas of Sources with Spitzer IRS Spectra $\left(\right.$ CASSIS $^{8}$, Lebouteiller et al. 2011, 2015) since a significant fraction of SyCAT sources ( 122 out of 351, i.e., 35\%) have mid-IR spectra already available.

It is worth mentioning that similar tests of the unification scenario were performed in the past by Mulchaey et al. (1994) for a sample of 116 Seyfert galaxies. They found that distributions of [O III] 15007 , infrared, and hard X-ray continuum are similar for Seyfert type 1 and 2. They also discovered a correlation between the ultraviolet continuum and emission-line fluxes in type 1 Seyfert galaxies, but not for type 2, consistent with the

8 https://cassis.sirtf.com 
idea that the central engine is responsible for powering the line emission, all properties are consistent with those expected in the dusty torus model.

As future perspectives, correlations between the mid-IR and the hard X-ray fluxes could be used to search for heavily obscured Seyfert galaxies, among unidentified hard X-ray sources seen by Swift-BAT and INTEGRAL. Having strong dust absorption, unknown Seyfert galaxies could not emit significantly in the optical energy range, but could show mid-IR to hard X-ray flux ratios similar to those listed in our Turin-SyCAT. We also expect to investigate the large-scale environments of Seyfert galaxies (Dultzin-Hacyan et al. 1999; Koulouridis et al. 2006; Villarroel \& Korn 2014; Jiang et al. 2016) using sources listed in Turin-SyCAT adopting the same statistical procedures described in Massaro et al. $(2019,2020)$ as an additional test for the unification scenario.

Finally, it is worth noting that we are already working on the second release of the Turin-SyCAT, planned for the end of 2021, to increase the number of Seyfert galaxies listed there to 1000 sources. The next release will be carried out including sources from SDSS and ROSAT surveys, thus not based on hard X-ray catalogs.

Acknowledgements. We thank the anonymous referee for useful comments that led to improvements in the paper. H.A.P.H and F.M. are in debt with Prof. D. Weedman for all useful discussions and wish to thank him for all extremely valuable comments and suggestions provided while carrying out this project. This work is supported by the "Departments of Excellence 2018 - 2022" Grant awarded by the Italian Ministry of Education, University and Research (MIUR) (L. 232/2016). H.A.P.-H acknowledges support from CONACyT program for Ph.D. studies. This work was partially supported from CONACyT research grant No. 280789. This investigation is supported by the National Aeronautics and Space Administration (NASA) grants GO9-20083X and GO021110X. A.P. acknowledges financial support from the Consorzio Interuniversitario per la fisica Spaziale (CIFS) under the agreement related to the grant MASF_CONTR_FIN_18_02. This research has made use of data obtained from the high-energy Astrophysics Science Archive Research Center (HEASARC) provided by NASA's Goddard Space Flight Center; the SIMBAD database operated at CDS, Strasbourg, France; the NASA/IPAC Extragalactic Database (NED) operated by the Jet Propulsion Laboratory, California Institute of Technology, under contract with the National Aeronautics and Space Administration. Part of this work is based on the NVSS (NRAO VLA Sky Survey): The National Radio Astronomy Observatory is operated by Associated Universities, Inc., under contract with the National Science Foundation and on the VLA low-frequency Sky Survey (VLSS). The Molonglo Observatory site manager, Duncan Campbell-Wilson, and the staff, Jeff Webb, Michael White and John Barry, are responsible for the smooth operation of Molonglo Observatory Synthesis Telescope (MOST) and the day-to-day observing programme of SUMSS. The SUMSS survey is dedicated to Michael Large whose expertise and vision made the project possible. The MOST is operated by the School of Physics with the support of the Australian Research Council and the Science Foundation for Physics within the University of Sydney. This publication makes use of data products from the Wide-field Infrared Survey Explorer, which is a joint project of the University of California, Los Angeles, and the Jet Propulsion Laboratory/California Institute of Technology, funded by the National Aeronautics and Space Administration. This publication makes use of data products from the Two Micron All Sky Survey, which is a joint project of the University of Massachusetts and the Infrared Processing and Analysis Center/California Institute of Technology, funded by the National Aeronautics and Space Administration and the National Science Foundation. Funding for the SDSS and SDSS-II has been provided by the Alfred P. Sloan Foundation, the Participating Institutions, the National Science Foundation, the US Department of Energy, the National Aeronautics and Space Administration, the Japanese Monbukagakusho, the Max Planck Society, and the Higher Education Funding Council for England. The SDSS Web Site is http://www.sdss.org/. The SDSS is managed by the Astrophysical Research Consortium for the Participating Institutions. The Participating Institutions are the American Museum of Natural History, Astrophysical Institute Potsdam, University of Basel, University of Cambridge, Case Western Reserve University, University of Chicago, Drexel University, Fermilab, the Institute for Advanced Study, the Japan Participation Group, Johns Hopkins University, the Joint Institute for Nuclear Astrophysics, the Kavli Institute for Particle Astrophysics and Cosmology, the Korean Scientist Group, the Chinese Academy of Sciences (LAMOST), Los Alamos National Laboratory, the Max-Planck-Institute for Astronomy (MPIA), the Max-Planck-Institute for Astrophysics (MPA), New Mexico State University, Ohio State University, University of Pittsburgh, University of Portsmouth, Princeton University, the United States Naval Observatory, and the University of Washington. This research has made use of the Palermo BAT catalog and database operated at INAF - IASF Palermo TOPCAT (http://www.star.bris.ac.uk/ mbt/topcat/) (Taylor 2005) for the preparation and manipulation of the tabular data and the images. The Aladin Java applet (http://aladin.u-strasbg.fr/aladin.gml) was used to create the finding charts reported in this paper (Bonnarel et al. 2000). It can be started from the CDS (Strasbourg - France), from the CFA (Harvard USA), from the ADAC (Tokyo - Japan), from the IUCAA (Pune - India), from the UKADC (Cambridge - UK), or from the CADC (Victoria - Canada).

\section{References}

Abazajian, K., Adelman-McCarthy, J. K., Agüeros, M. A., et al. 2005, AJ, 129, 1755

Abdollahi, S., Acero, F., Ackermann, M., et al. 2020, ApJS, 247, 33

Ackermann, M., Ajello, M., Allafort, A., et al. 2012, ApJ, 755, 164

Adams, T. F. 1977, ApJS, 33, 19

Aguado, D. S., Ahumada, R., Almeida, A., et al. 2019, ApJS, 240, 23

Ajello, M., Romani, R. W., Gasparrini, D., et al. 2014, ApJ, 780, 73

Alonso-Herrero, A., Ramos Almeida, C., Mason, R., et al. 2011, ApJ, 736, 82

Alonso-Herrero, A., García-Burillo, S., Pereira-Santaella, M., et al. 2019, A\&A, 628, A65

Álvarez Crespo, N., Massaro, F., Milisavljevic, D., et al. 2016, AJ, 151, 95

Antonucci, R. 1993, ARA\&A, 31, 473

Antonucci, R. R. J., \& Miller, J. S. 1985, ApJ, 297, 621

Aretxaga, I., Joguet, B., Kunth, D., Melnick, J., \& Terlevich, R. J. 1999, ApJ, 519,123

Arsioli, B., Fraga, B., Giommi, P., et al. 2015, A\&A, 579, A34

Ashby, M. L. N., Stern, D., Brodwin, M., et al. 2009, ApJ, 701, 428

Asmus, D., Gandhi, P., Hönig, S. F., et al. 2015, MNRAS, 454, 766

Baldwin, J. A., Phillips, M. M., \& Terlevich, R. 1981, PASP, 93, 5

Baloković, M., Smolčić, V., Ivezić, Ž., et al. 2012, ApJ, 759, 30

Barnothy, J. M., \& Barnothy, M. F. 1968, AJ, 73, 912

Baumgartner, W. H., Tueller, J., Markwardt, C. B., et al. 2013, ApJS, 207, 19

Berlin, A., \& Hooper, D. 2014, Phys. Rev. D, 89, 016014

Bernieri, E., Campana, R., Massaro, E., et al. 2013, A\&A, 551, L5

Beasley, A. J., Gordon, D., Peck, A. B., et al. 2002, ApJS, 141, 13

Beckmann, V., Soldi, S., Ricci, C., et al. 2009, A\&A, 505, 417

Bennett, C. L., Larson, D., Weiland, J. L., \& Hinshaw, G. 2014, ApJ, 794, 135

Bianchi, S., Matt, G., Balestra, I., et al. 2004, A\&A, 422, 65

Bianchi, S., Guainazzi, M., \& Chiaberge, M. 2006, A\&A, 448, 499

Bianchi, S., Guainazzi, M., Matt, G., et al. 2009a, A\&A, 495, 421

Bianchi, S., Piconcelli, E., Chiaberge, M., et al. 2009b, ApJ, 695, 781

Bird, A. J., Malizia, A., Bazzano, A., et al. 2007, ApJS, 170, 175

Bonnarel, F., Fernique, P., Bienaymé, O., et al. 2000, A\&AS, 143, 33

Bontempi, P., Giroletti, M., Panessa, F., et al. 2012, MNRAS, 426, 588

Boroson, T. A., \& Green, R. F. 1992, ApJS, 80, 109

Burbidge, G. R., \& Hoyle, F. 1968, AJ, 73, 907

Burbidge, E. M., Burbidge, G. R., \& Prendergast, K. H. 1959, ApJ, 130, 26

Burbidge, G. R., Burbidge, E. M., \& Sandage, A. R. 1963, Rev. Mod. Phys., 35, 947

Burtscher, L., Davies, R. I., Graciá-Carpio, J., et al. 2016, A\&A, 586, A28

Calzetti, D. 2011, EAS Publ. Ser., 133

Capetti, A., Massaro, F., \& Baldi, R. D. 2017a, A\&A, 601, A81

Capetti, A., Massaro, F., \& Baldi, R. D. 2017b, A\&A, 598, A49

Chiaraluce, E., Bruni, G., Panessa, F., et al. 2019, MNRAS, 485, 3185

Cid Fernandes, R., Heckman, T., Schmitt, H., et al. 2001, ApJ, 558, 81

Colless, M., Dalton, G., Maddox, S., et al. 2001, MNRAS, 328, 1039

Collin-Souffrin, S., Alloin, D., \& Andrillat, Y. 1973, A\&A, 22, 343

Corral, A., Della Ceca, R., Caccianiga, A., et al. 2011, A\&A, 530, A42

Contini, M., \& Viegas, S. M. 2000, ApJ, 535, 721

Croom, S. M., Smith, R. J., Boyle, B. J., et al. 2004, MNRAS, 349, 1397

Cusumano, G., La Parola, V., Segreto, A., et al. 2010, A\&A, 524, A64

Cusumano, G., Segreto, A., La Parola, V., et al. 2014, Proceedings of Swift: 10

Years of Discovery (SWIFT), 10, 132

D'Abrusco, R., Massaro, F., Ajello, M., et al. 2012, ApJ, 748, 68

D'Abrusco, R., Massaro, F., Paggi, A., et al. 2013, ApJS, 206, 12

D’Abrusco, R., Massaro, F., Paggi, A., et al. 2014, ApJS, 215, 14

D’Abrusco, R., Álvarez Crespo, N., Massaro, F., et al. 2019, ApJS, 242, 4

Dahari, O. 1984, AJ, 89, 966

Davies, R. I., Müller Sánchez, F., Genzel, R., et al. 2007, ApJ, 671, 1388

de Grijp, M. H. K., Keel, W. C., Miley, G. K., et al. 1992, A\&AS, 96, 389

de Menezes, R., Peña-Herazo, H. A., Marchesini, E. J., et al. 2019, A\&A, 630,

A55 
Denney, K. D., De Rosa, G., Croxall, K., et al. 2014, ApJ, 796, 134 De Robertis, M. M., Hayhoe, K., \& Yee, H. K. C. 1998, ApJS, 115, 163 Diamond-Stanic, A. M., \& Rieke, G. H. 2012, ApJ, 746, 168 Dressler, A., \& Shectman, S. A. 1988, AJ, 95, 284

Dressler, A., Thompson, I. B., \& Shectman, S. A. 1985, ApJ, 288, 481 Dultzin-Hacyan, D., Krongold, Y., Fuentes-Guridi, I., et al. 1999, ApJ, 513, L111 Edge, D. O., Shakeshaft, J. R., McAdam, W. B., et al. 1959, MmRAS, 68, 37

Efstathiou, A., \& Rowan-Robinson, M. 1995, MNRAS, 273, 649

Esparza-Arredondo, D., González-Martín, O., Dultzin, D., et al. 2018, ApJ, 859. 124

Fath, E. A. 1909, LicOB, 149, 71

Fabian, A. C., Guilbert, P. W., Arnaud, K. A., et al. 1986, MNRAS, 218, 457

Ferland, G. J., \& Netzer, H. 1983, ApJ, 264, 105

Feruglio, C., Fabbiano, G., Bischetti, M., et al. 2020, ApJ, 890, 29

Fey, A. L., Gordon, D., Jacobs, C. S., et al. 2015, AJ, 150, 58

Fomalont, E. B., Petrov, L., MacMillan, D. S., et al. 2003, AJ, 126, 2562

Fuentes-Williams, T., \& Stocke, J. T. 1988, AJ, 96, 1235

Fukugita, M., Ichikawa, T., Gunn, J. E., et al. 1996, AJ, 111, 1748

Gallimore, J. F., Yzaguirre, A., Jakoboski, J., et al. 2010, ApJS, 187, 172

Gandhi, P., Horst, H., Smette, A., et al. 2009, A\&A, 502, 457

García-Burillo, S., Combes, F., Usero, A., et al. 2014, A\&A, 567, A125

García-Bernete, I., Ramos Almeida, C., Acosta-Pulido, J. A., et al. 2016, MNRAS, 463, 3531

Gaskell, M. 2014, Nat. Phys, 10, 414

George, I. M., \& Fabian, A. C. 1991, MNRAS, 249, 352

Gliozzi, M., \& Williams, J. K. 2020, MNRAS, 491, 532

González-Martín, O., Masegosa, J., García-Bernete, I., et al. 2019, ApJ, 884, 11 Gordon, Y. A., Owers, M. S., Pimbblet, K. A., et al. 2017, MNRAS, 465, 2671 Gower, J. F. R., Scott, P. F., \& Wills, D. 1967, MmRAS, 71, 49

Green, R. F. 1976, PASP, 88, 665

Green, R. F., Schmidt, M., \& Liebert, J. 1986, ApJS, 61, 305

Haardt, F., \& Maraschi, L. 1991, ApJ, 380, L51

Hao, L., Weedman, D. W., Spoon, H. W. W., et al. 2007, ApJ, 655, L77

Haro, G. 1956, Bol. Obs. Tonantzintla Tacubaya, 2, 8

Hayashida, M., Stawarz, Ł., Cheung, C. C., et al. 2013, ApJ, 779, 131

Heckman, T. M. 1978, PASP, 90, 241

Heckman, T. M. 1980, A\&A, 500, 187

Heckman, T. M., \& Best, P. N. 2014, ARA\&A, 52, 589

Ho, L. C. 2008, ARA\&A, 46, 475

Ho, L. C., \& Ulvestad, J. S. 2001, ApJS, 133, 77

Ho, L. C., Filippenko, A. V., \& Sargent, W. L. 1995, ApJS, 98, 477

Ho, L. C., Filippenko, A. V., Sargent, W. L. W., \& Peng, C. Y. 1997, ApJS, 112, 391

Ho, L. C., Filippenko, A. V., \& Sargent, W. L. W. 2003, ApJ, 583, 159

Hoyle, F., \& Fowler, W. A. 1963, Nature, 197, 533

Hubble, E. P. 1926, ApJ, 64, 321

Huchra, J., \& Burg, R. 1992, ApJ, 393, 90

Huchra, J. P., Vogeley, M. S., \& Geller, M. J. 1999, ApJS, 121, 287

Humason, M. L. 1932, PASP, 44, 267

Ichikawa, K., Ricci, C., Ueda, Y., et al. 2017, ApJ, 835, 74

Ichikawa, K., Ricci, C., Ueda, Y., et al. 2019, ApJ, 870, 31

Jiang, N., Wang, H., Mo, H., et al. 2016, ApJ, 832, 111

Jensen, J. J., Hönig, S. F., Rakshit, S., et al. 2017, MNRAS, 470, 3071

Jones, D. H., Saunders, W., Colless, M., et al. 2004, MNRAS, 355, 747

Kauffmann, G., Heckman, T. M., Tremonti, C., et al. 2003, MNRAS, 346, 1055

Keel, W. C. 1980, AJ, 85, 198

Kewley, L. J., Dopita, M. A., Sutherland, R. S., et al. 2001, ApJ, 556, 121

Kewley, L. J., Groves, B., Kauffmann, G., et al. 2006, MNRAS, 372, 961

Khachikian, E. E., \& Weedman, D. W. 1971, Astrofizika, 7, 389

Khachikian, E. Y., \& Weedman, D. W. 1974, ApJ, 192, 581

Koski, A. T. 1978, ApJ, 223, 56

Koss, M., Trakhtenbrot, B., Ricci, C., et al. 2017, ApJ, 850, 74

Kotilainen, J. K., \& Ward, M. J. 1994, MNRAS, 266, 953

Koulouridis, E., Plionis, M., Chavushyan, V., et al. 2006, ApJ, 639, 37

LaMassa, S. M., Heckman, T. M., Ptak, A., et al. 2012, ApJ, 758, 1

LaMassa, S. M., Cales, S., Moran, E. C., et al. 2015, ApJ, 800, 144

Landoni, M., Massaro, F., Paggi, A., et al. 2015, AJ, 149, 163

Laurikainen, E., \& Salo, H. 1995, A\&A, 293, 683

Lebouteiller, V., Barry, D. J., Spoon, H. W. W., et al. 2011, ApJS, 196, 8

Lebouteiller, V., Barry, D. J., Goes, C., et al. 2015, ApJS, 218, 21

Lintott, C. J., Schawinski, K., Slosar, A., et al. 2008, MNRAS, 389, 1179

Lipovetsky, V. A., Neizvestnyj, S. I., \& Neizvestnaya, O. M. 1987, Soobshcheniya Spetsial'noj Astrofizicheskoj Observatorii, 55

Lipovetsky, V. A., Neizvestny, S. I., \& Neizvestnaya, O. M. 1988, Soobshcheniya Spetsial'noj Astrofizicheskoj Observatorii, 55, 5

Lutz, D., Maiolino, R., Spoon, H. W. W., et al. 2004, A\&A, 418, 465

MacKenty, J. W. 1989, ApJ, 343, 125

MacKenty, J. W. 1990, ApJS, 72, 231
Maiolino, R., \& Rieke, G. H. 1995, ApJ, 454, 95

Maiolino, R., Salvati, M., Bassani, L., et al. 1998, A\&A, 338, 781

Mantovani, G., Nandra, K., \& Ponti, G. 2016, MNRAS, 458, 4198

Marchesi, S., Kaur, A., \& Ajello, M. 2018, AJ, 156, 212

Marchesini, E. J., Masetti, N., Chavushyan, V., et al. 2016, A\&A, 596, A10

Marchesini, E. J., Peña-Herazo, H. A., Álvarez Crespo, N., et al. 2019a, Ap\&SS, 364, 5

Marchesini, E. J., Masetti, N., Palazzi, E., et al. 2019b, Ap\&SS, 364, 153

Markarian, B. E. 1967, Astrofizika, 3

Markarian, B. E., Lipovetsky, V. A., Stepanian, J. A., et al. 1989, Soobshcheniya

Spetsial'noj Astrofizicheskoj Observatorii, 62, 5

Markowitz, A., Reeves, J. N., Miniutti, G., et al. 2008, PASJ, 60, S277

Masetti, N., Palazzi, E., Bassani, L., et al. 2004, A\&A, 426, L41

Masetti, N., Mason, E., Bassani, L., et al. 2006, A\&A, 448, 547

Masetti, N., Mason, E., Morelli, L., et al. 2008, A\&A, 482, 113

Masetti, N., Parisi, P., Palazzi, E., et al. 2009, A\&A, 495, 121

Masetti, N., Parisi, P., Palazzi, E., et al. 2010, A\&A, 519, A96

Masetti, N., Parisi, P., Jiménez-Bailón, E., et al. 2012, A\&A, 538, A123

Masetti, N., Parisi, P., Palazzi, E., et al. 2013, A\&A, 556, A120

Mason, R. E., Levenson, N. A., Shi, Y., et al. 2009, ApJ, 693, L136

Massaro, F., \& D'Abrusco, R. 2016, ApJ, 827, 67

Massaro, F., Bianchi, S., Matt, G., et al. 2006, A\&A, 455, 153

Massaro, E., Giommi, P., Leto, C., et al. 2009, A\&A, 495, 691

Massaro, F., D’Abrusco, R., Ajello, M., et al. 2011, ApJ, 740, L48

Massaro, F., D'Abrusco, R., Tosti, G., et al. 2012a, ApJ, 750, 138

Massaro, F., D’Abrusco, R., Tosti, G., et al. 2012b, ApJ, 752, 61

Massaro, F., D’Abrusco, R., Paggi, A., et al. 2013a, ApJS, 206, 13

Massaro, F., D'Abrusco, R., Paggi, A., et al. 2013b, ApJS, 209, 10

Massaro, F., Masetti, N., D’Abrusco, R., et al. 2014, AJ, 148, 66

Massaro, E., Maselli, A., Leto, C., et al. 2015a, Ap\&SS, 357, 75

Massaro, F., D’Abrusco, R., Landoni, M., et al. 2015b, ApJS, 217, 2

Massaro, F., Landoni, M., D'Abrusco, R., et al. 2015c, A\&A, 575, A124

Massaro, F., Álvarez Crespo, N., D’Abrusco, R., et al. 2016, Ap\&SS, 361, 337

Massaro, F., Álvarez-Crespo, N., Capetti, A., et al. 2019, ApJS, 240, 20

Massaro, F., Capetti, A., Paggi, A., et al. 2020, ApJS, 247, 71

Matt, G. 1997, Mem. Soc. Astron. It., 68, 127

Matt, G., Brandt, W. N., \& Fabian, A. C. 1996, MNRAS, 280, 823

Matt, G., Guainazzi, M., \& Maiolino, R. 2003, MNRAS, 342, 422

Mayall, N. U. 1934, PASP, 46, 134

Mehdipour, M., Branduardi-Raymont, G., Kaastra, J. S., et al. 2011, A\&A, 534, A39

Menezes, R. B., da Silva, P., \& Steiner, J. E. 2018, MNRAS, 473, 2198

Merloni, A., Bongiorno, A., Brusa, M., et al. 2014, MNRAS, 437, 3550

Mor, R., Netzer, H., \& Elitzur, M. 2009, ApJ, 705, 298

Morganti, R., Oosterloo, T., Oonk, J. B. R., et al. 2015, A\&A, 580, A1

Mulchaey, J. S., Koratkar, A., Ward, M. J., et al. 1994, ApJ, 436, 586

Mushotzky, R. F., Marshall, F. E., Boldt, E. A., et al. 1980, ApJ, 235, 377

Nandra, K., \& Pounds, K. A. 1994, MNRAS, 268, 405

Nenkova, M., Sirocky, M. M., Nikutta, R., et al. 2008, ApJ, 685, 160

Oh, K., Koss, M., Markwardt, C. B., et al. 2018, ApJS, 235, 4

Ordovás-Pascual, I., Mateos, S., Carrera, F. J., et al. 2017, MNRAS, 469, 693

Osterbrock, D. E. 1981, ApJ, 249, 462

Osterbrock, D. E., \& Martel, A. 1993, ApJ, 414, 552

Osterbrock, D. E., \& Parker, R. A. R. 1965, ApJ, 141, 892

Osterbrock, D. E., \& Pogge, R. W. 1985, ApJ, 297, 166

Pacholczyk, A. G., \& Weymann, R. 1968, AJ, 73, 836

Paggi, A., Milisavljevic, D., Masetti, N., et al. 2014, AJ, 147, 112

Paiano, S., Landoni, M., Falomo, R., et al. 2017, ApJ, 844, 120

Paiano, S., Falomo, R., Treves, A., et al. 2019, ApJ, 871, 162

Panessa, F., \& Bassani, L. 2002, A\&A, 394, 435

Panessa, F., Bassani, L., Cappi, M., et al. 2006, A\&A, 455, 173

Panessa, F., Baldi, R. D., Laor, A., et al. 2019, Nat. Astron., 3, 387

Pappa, A., Georgantopoulos, I., Stewart, G. C., et al. 2001, MNRAS, 326, 995

Parisi, P., Masetti, N., Jiménez-Bailón, E., et al. 2012, A\&A, 545, A101

Patrick, A. R., Reeves, J. N., Porquet, D., et al. 2012, MNRAS, 426, 2522

Peeters, E., Spoon, H. W. W., \& Tielens, A. G. G. M. 2004, ApJ, 613, 986

Peña-Herazo, H. A., Marchesini, E. J., Álvarez Crespo, N., et al. 2017, Ap\&SS, 362, 228

Peña-Herazo, H. A., Massaro, F., Chavushyan, V., et al. 2019, Ap\&SS, 364, 85

Petrosian, A. R. 1982, Astrofizika, 18, 548

Pietsch, W., Bischoff, K., Boller, T., et al. 1998, A\&A, 333, 48

Pilkington, J. D. H., \& Scott, J. F. 1965, MmRAS, 69, 183

Pogge, R. W. 1988, ApJ, 332, 702

Predehl, P. 2017, Astron. Nachr., 338, 159

Rafanelli, P., \& Schulz, H. 1991, Astron. Nachr., 312, 167

Rafanelli, P., Violato, M., \& Baruffolo, A. 1995, AJ, 109, 1546 
Raimundo, S. I., Vestergaard, M., Koay, J. Y., et al. 2019, MNRAS, 486, 123 Ramos Almeida, C., Pérez García, A. M., Acosta-Pulido, J. A., et al. 2007, AJ, 134, 2006

Ricci, C., Ueda, Y., Koss, M. J., et al. 2015, ApJ, 815, L13

Rieke, G. H. 1978, ApJ, 226, 550

Risaliti, G., Maiolino, R., \& Salvati, M. 1999, ApJ, 522, 157

Roche, P. F., Packham, C., Aitken, D. K., et al. 2007, MNRAS, 375, 99

Rojas, A. F., Masetti, N., Minniti, D., et al. 2017, A\&A, 602, A124

Ruschel-Dutra, D., Pastoriza, M., Riffel, R., et al. 2014, MNRAS, 438, 3434

Ruschel-Dutra, D., Rodríguez Espinosa, J. M., González Martín, O., et al. 2017, MNRAS, 466, 3353

Salvato, M., \& Rafanelli, P. 1997, Astron. Nachr., 318, 237

Sandage, A., \& Tammann, G. A. 1987, A revised Shapley-Ames Catalog of bright galaxies (Carnegie Institution of Washington Publication)

Sani, E., Lutz, D., Risaliti, G., et al. 2010, MNRAS, 403, 1246

Sargent, W. L. W. 1970, ApJ, 160, 405

Sazonov, S., Willner, S. P., Goulding, A. D., et al. 2012, ApJ, 757, 181

Schawinski, K., Thomas, D., Sarzi, M., et al. 2007, MNRAS, 382, 1415

Schmidt, M. 1963, Nature, 197, 1040

Schmidt, M., \& Green, R. F. 1983, ApJ, 269, 352

Sebastian, B., Kharb, P., O'Dea, C. P., et al. 2020, MNRAS, 499, 334

Segreto, A., Cusumano, G., Ferrigno, C., et al. 2010, A\&A, 510, A47

Seyfert, C. K. 1943, ApJ, 97, 28

Shappee, B. J., Prieto, J. L., Grupe, D., et al. 2014, ApJ, 788, 48

Shi, Y., Rieke, G. H., Smith, P., et al. 2010, ApJ, 714, 115

Siebenmorgen, R., Heymann, F., \& Efstathiou, A. 2015, A\&A, 583, A120

Singh, V., Shastri, P., \& Risaliti, G. 2011, A\&A, 532, A84

Simkin, S. M., Su, H. J., \& Schwarz, M. P. 1980, ApJ, 237, 404

Slipher, V. M. 1917, Lowell Obs. Bull., 3, 59
Souchay, J., Andrei, A. H., Barache, C., et al. 2009, A\&A, 494, 799

Stasińska, G. 1984, A\&A, 135, 341

Stasińska, G., Cid Fernandes, R., Mateus, A., et al. 2006, MNRAS, 371, 972 Stern, D., Assef, R. J., Benford, D. J., et al. 2012, ApJ, 753, 30

Storchi-Bergmann, T., Fernandes, R. C., \& Schmitt, H. R. 1998, ApJ, 501, 94

Sturm, E., Schweitzer, M., Lutz, D., et al. 2005, ApJ, 629, L21

Taylor, M. B. 2005, ASPC, 347, 29

Treister, E., Castander, F. J., Maccarone, T. J., et al. 2005, ApJ, 621, 104

Tristram, K. R. W., Meisenheimer, K., Jaffe, W., et al. 2007, A\&A, 474, 837

Tueller, J., Mushotzky, R. F., Barthelmy, S., et al. 2008, ApJ, 681, 113

Urry, C. M., \& Padovani, P. 1995, PASP, 107, 803

Veilleux, S., \& Osterbrock, D. E. 1987, ApJS, 63, 295

Villarroel, B., \& Korn, A. J. 2014, Nat. Phys., 10, 417

Veron-Cetty, M.-P., \& Veron, P. 1989, ESO Scientific Report

Véron-Cetty, M.-P., \& Véron, P. 2006, A\&A, 455, 773

Véron-Cetty, M.-P., \& Véron, P. 2010, A\&A, 518, A10

Voges, W., Aschenbach, B., Boller, T., et al. 1999, A\&A, 349, 389

Voges, W., Aschenbach, B., Boller, T., et al. 2000, VizieR Online Data Catalog:IX/29

Walter, R., \& Fink, H. H. 1993, A\&A, 274, 105

Weedman, D. W. 1976, QJRAS, 17, 22

Weedman, D. W. 1977, ARA\&A, 15, 69

Wojaczyński, R., Niedźwiecki, A., Xie, F.-G., et al. 2015, A\&A, 584, A20

Woltjer, L. 1959, ApJ, 130, 38

Wright, E. L., Eisenhardt, P. R. M., Mainzer, A. K., et al. 2010, AJ, 140, 1868 Xanthopoulos, E. 1996, MNRAS, 280, 6

Xie, Y., Li, A., \& Hao, L. 2017, ApJS, 228, 6

Yee, H. K. 1983, ApJ, 272, 473

Zechlin, H.-S., \& Horns, D. 2012, J. Cosmol. Astropart. Phys., 2012, 050 


\section{Appendix A: SyCAT tables}

Here we report the first five rows for each table of the TurinSyCAT as described in the following.

In Table A.1 there are all 351 Turin-SyCAT sources with (i) their unique catalog identification number (ID); (ii) their SyCAT name; (iii,iv,v) radio names from the SUMSS and NVSS catalogs if a counterpart is present; (vi) name of the unique WISE counterpart as well as (vii) that in the optical from the Pan-STARRS if in the footprint above a declination of $\sim 33^{\circ}$; and (viii,ix,x,xi) X-ray names from ROSAT, 3PBC, BAT105, and IBIS4CAT, respectively. This table is extremely important to carry out cross-matches easily with other catalogs, surveys, and/or samples.

Table A.2 reports the main properties of the catalog as (i) unique catalog ID; (ii) SyCAT name; (iii) name of the mid-IR WISE counterpart; (iv,v) WISE coordinates; (vi,vii) redshift and luminosity distance computed according to the chosen cosmology, respectively; (viii) the type 1 or type 2 classification provided by our analysis; (ix) the reference for the redshift; and (x,xi) the class indicated in the literature and the reference for it.

In Table A.3 we provide all the information regarding the mid-IR properties, such as all WISE and 2MASS magnitudes, including their uncertainties, and the $\mathrm{L}_{W 1}$ luminosity used to avoid quasar selection compared to the $10^{11} L_{\odot}$ threshold.

The main optical information (i.e., magnitudes and spectral classification, if any) concerning the cross-matches obtained with the SDSS, and the Pan-STARRS databases are reported in Table A.4, together with their (1) unique ID; (2) SyCAT name; (3) name in Pan-STARRS survey. Columns(4), (5), (6), (7), and (8) are the PSF magnitudes from Pan-STARRS in $g, r, i, z$, and $y$ bands and their uncertainties in parentheses. The following columns contain SDSS information: (9) flag of presence in SDSS; (10) magnitude from SDSS in u band and its $1 \sigma$ uncertainty in parentheses; (11) spectroscopic redshift; (12) automatic classification; and (13) automatic subclassification. This information is provided only for Seyfert galaxies lying in the PanSTARRS footprint.

Finally, in Table A.5, we report all the parameters related to the X-ray counterparts of the Turin-SyCAT sources collected from the ROSAT, 3PBC, BAT105, and IBSI4CAT catalogs. This information is shown in the table only for those sources having at least one X-ray counterpart in one of the surveys previously indicated and again the (i) unique ID; (ii) SyCAT name; (iii) name of the mid-IR WISE counterpart are in the first three columns.

Table A.1. All Turin-SyCAT sources with names of their counterparts in the catalogs/surveys explored (first five rows, full table available at the CDS)

\begin{tabular}{|c|c|c|c|c|c|c|c|c|c|}
\hline (1) & $\begin{array}{l}\text { SyCAT } \\
\text { name } \\
\text { (2) }\end{array}$ & $\begin{array}{l}\text { SUMSS } \\
\text { name } \\
\text { (3) }\end{array}$ & $\begin{array}{l}\text { NVSS } \\
\text { name } \\
(4)\end{array}$ & $\begin{array}{l}\text { WISE } \\
\text { name } \\
(5)\end{array}$ & $\begin{array}{l}\text { Pan-STARRS } \\
\text { PSO name } \\
\text { (6) }\end{array}$ & $\begin{array}{l}\text { ROSAT } \\
\text { name } \\
\text { (7) }\end{array}$ & $\begin{array}{l}3 \mathrm{PBC} \\
\text { name } \\
(8)\end{array}$ & $\begin{array}{l}\text { BAT105 } \\
\text { Swift name } \\
\text { (9) }\end{array}$ & $\begin{array}{l}\text { IBIS4CAT } \\
\text { IGR name } \\
(10)\end{array}$ \\
\hline 1 & SY1 J0002+0322 & & \multirow{5}{*}{ NVSSJ000619+201210 } & $\mathrm{J} 000226.41+032107.0$ & PSO J000226.415+032106.991 & \multirow[t]{4}{*}{ J000226.6+032105 } & 3РВC J0002.5+0322 & SWIFT J0002.5+0323 & \multirow{3}{*}{ IGR J00040+7020 } \\
\hline 2 & SY2 J0004+7020 & & & J000401.97+701918.2 & PSO J000401.990+701918.250 & & 3РBC J0004.0+7018 & SWIFT J0005.0+7021 & \\
\hline 3 & SY1 J0006+2013 & & & J000619.53+201210.6 & PSO J000619.529+201210.662 & & 3РBC J0006.3+2012 & SWIFT J0006.2+2012 & \\
\hline 4 & SY2 J0025+6821 & & & J002532.37+682144.9 & PSO J002532.313+682145.249 & & 3РBC J0025.5+6822 & SWIFT J0025.8+6818 & IGR J00256+6821 \\
\hline 5 & SY1 J0025-1859 & & & J002542.34-190010.1 & PSO J002542.349-190010.366 & J002542.6-190009 & 3РBC J0025.6-1859 & & \\
\hline
\end{tabular}

Column description: (1) Unique catalog identified (ID); (2) SyCAT name; (3) name in SUMSS; (4) name in NVSS; (5) name in WISE; (6) name in Pan-STARRS; (7) name in ROSAT; (8) name in 3PBC; (9) name in BAT105; (10) name in IBIS4CAT. 

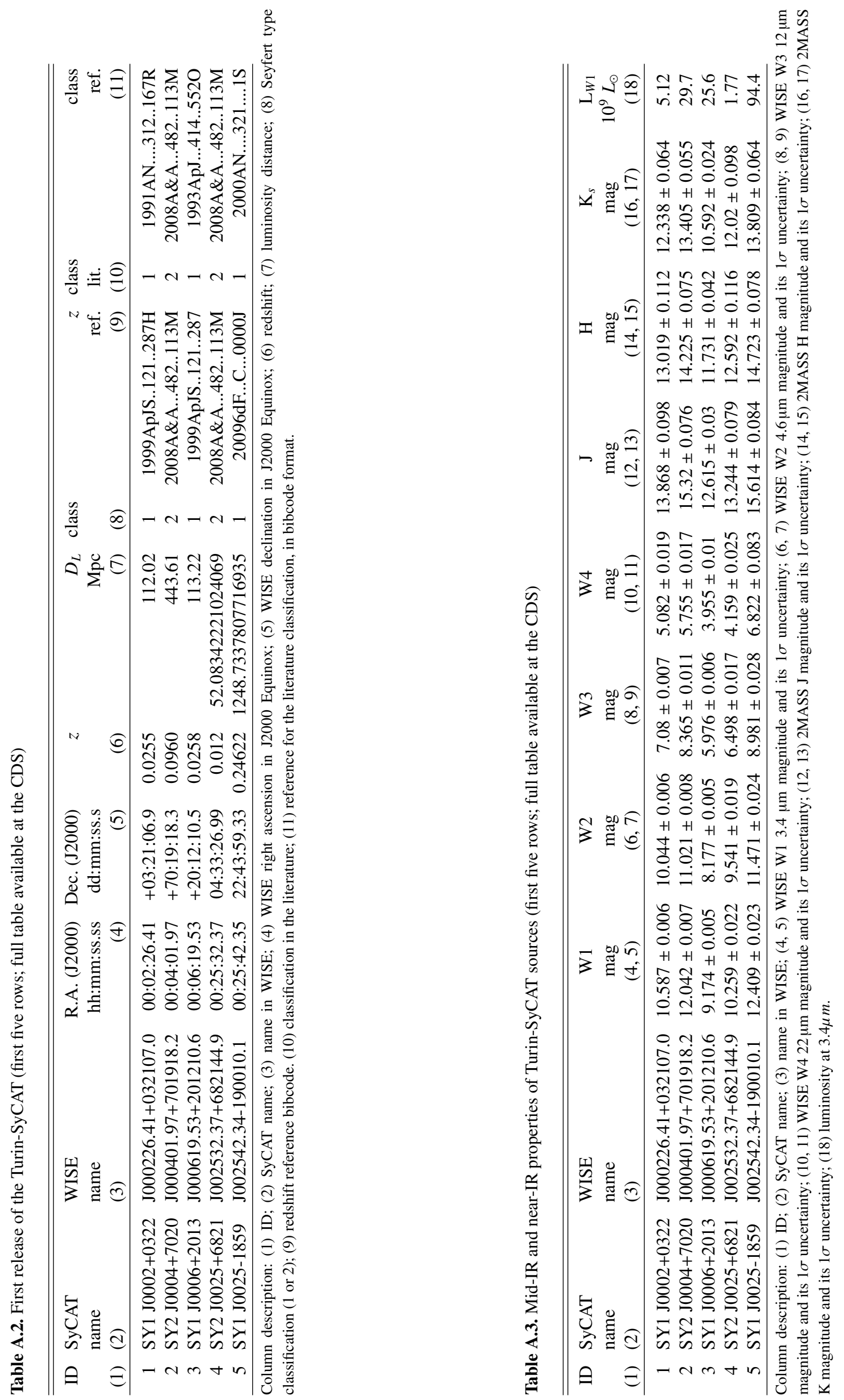
H. A. Peña-Herazo et al.: Turin-SyCAT: A multifrequency catalog of Seyfert galaxies
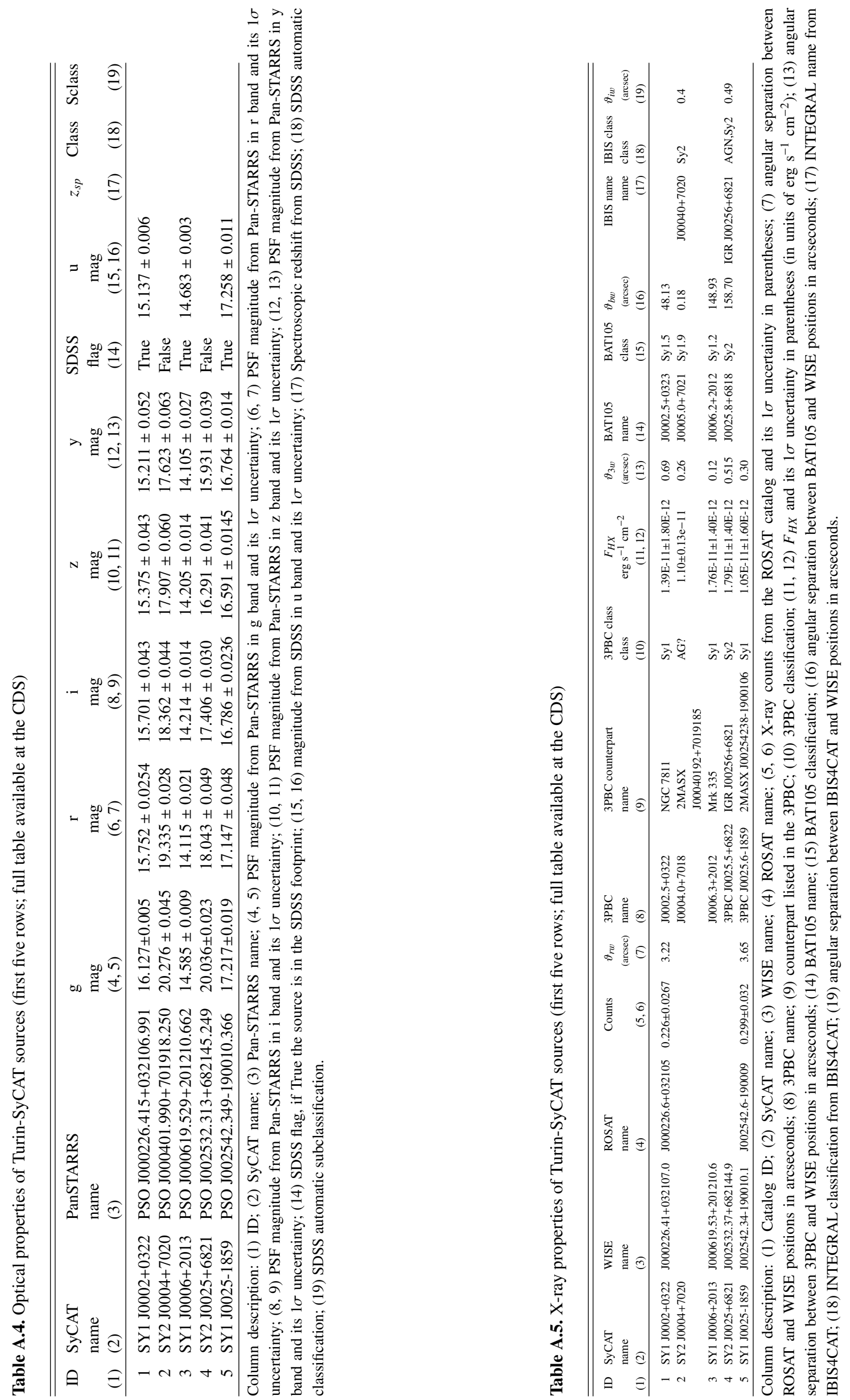


\section{Appendix B: Color dependence on redshift}

In this appendix we compare the dependence on redshift in the WISE color-color space for both Seyfert types. We present in Figures B.1 to B.6 the WISE colors w1-w2 versus w2-w3, and w3-w4 versus w2w3, as in Figure 7, but for different redshifts bins to show that the behavior of the two populations does not change with redshift. Up to redshift 0.1 , where we have type 2, both populations are clearly separated in the w1-w2 versus w2w3 color-color plot.

In Figure B.7 we explore the WISE color-color space for the subsample of Seyfert galaxies above the $90 \%$ identification fraction included in 3PBC.
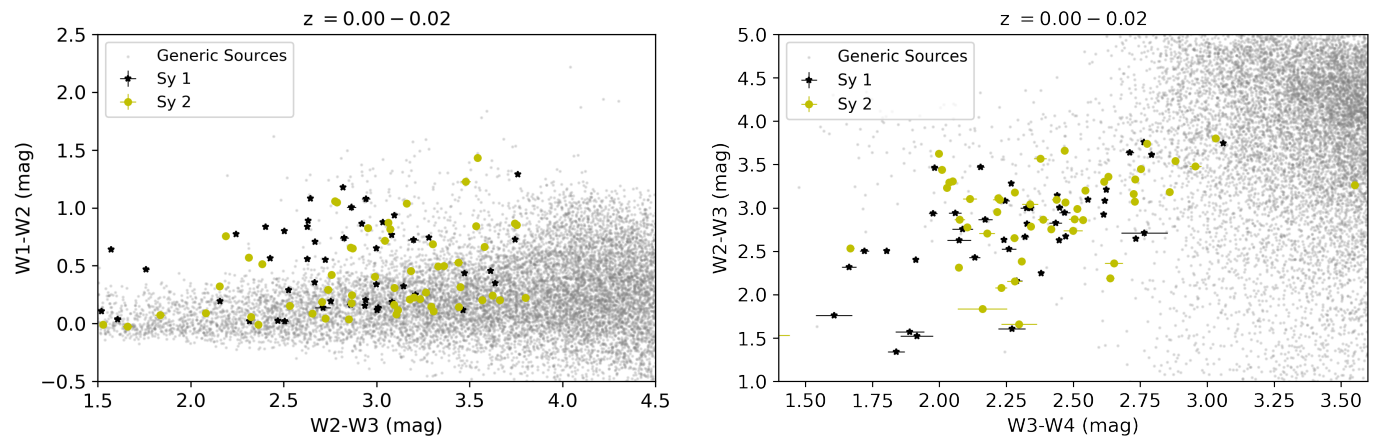

Fig. B.1. Color-color diagrams based on the WISE magnitudes comparing Seyfert galaxies of type 1 (black) and type 2 (yellow) with generic mid-IR sources selected in a random region of the sky (see Massaro et al. 2011, 2013a,b, 2016, for a similar analysis). Seyfert galaxies of type 2 appear to be redder than those of type 1, and are separated in the w1-w2 vs. w2-w3 color-color plot in the lower left corner.
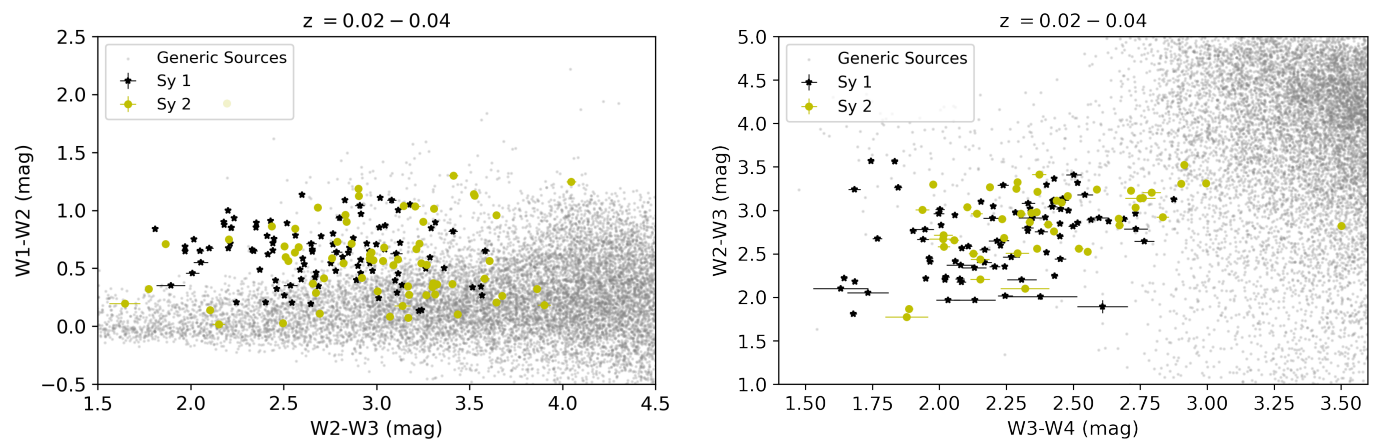

Fig. B.2. Same as Figure B.1, but for sources in the redshift range of 0.02 to 0.04 .
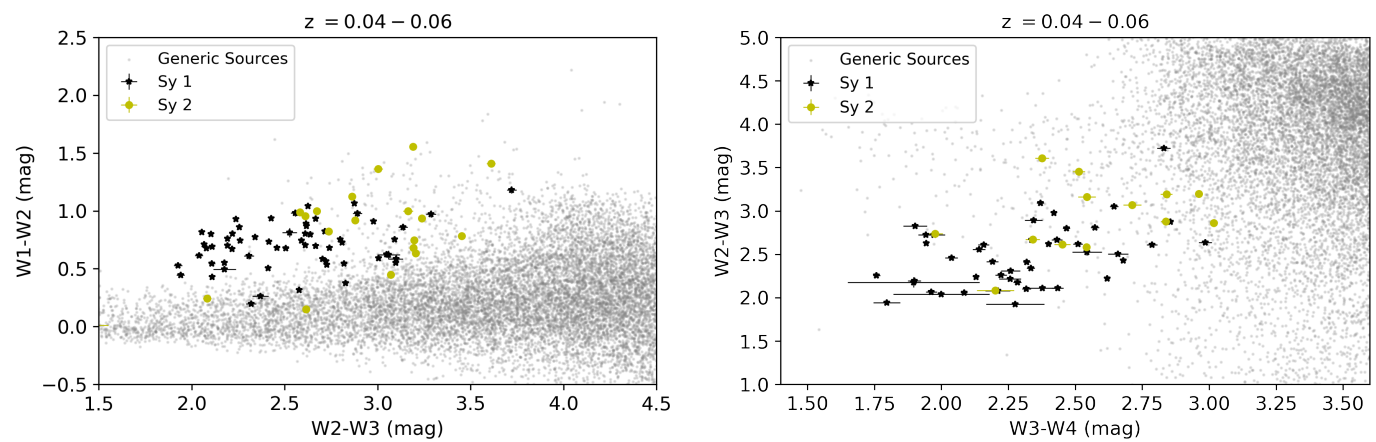

Fig. B.3. Same as Figure B.1, but for sources in the redshift range of 0.04 to 0.06 . 
H. A. Peña-Herazo et al.: Turin-SyCAT: A multifrequency catalog of Seyfert galaxies
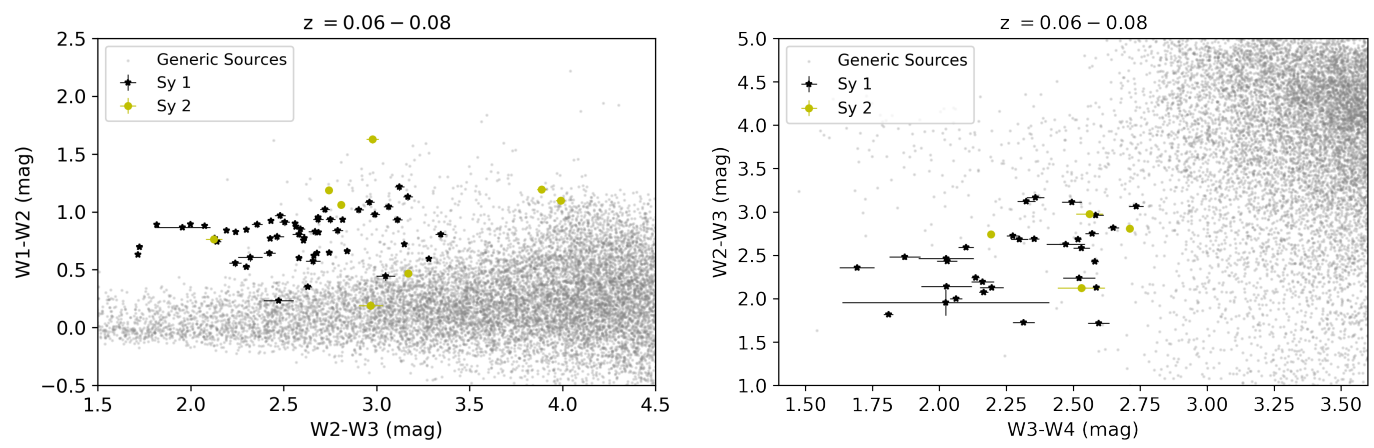

Fig. B.4. Same as Figure B.1, but for sources in the redshift range of 0.06 to 0.08 .
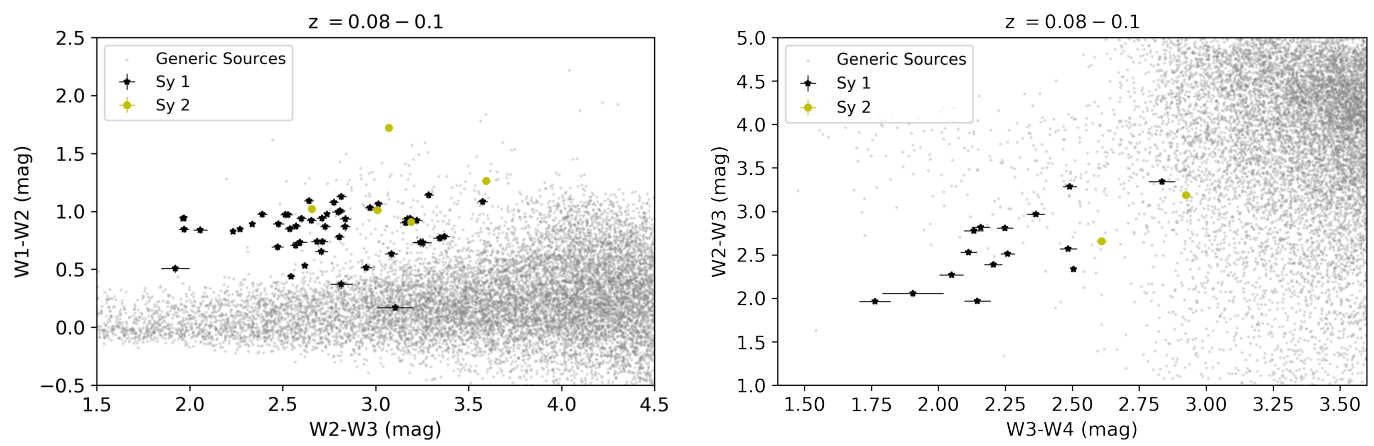

Fig. B.5. Same as Figure B.1, but for sources in the redshift range of 0.08 to 0.1 .
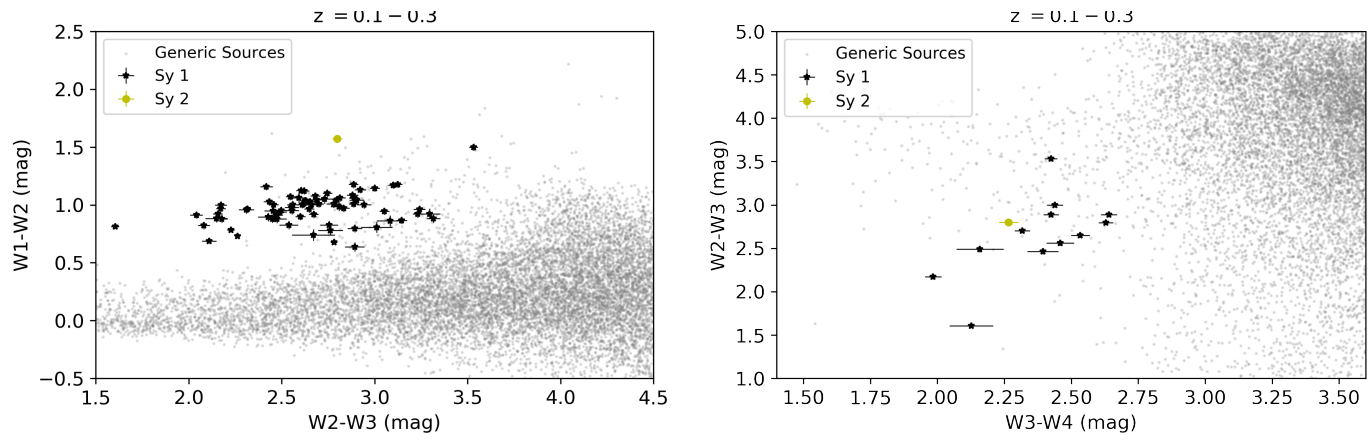

Fig. B.6. Same as Figure B.1, but for sources in the redshift range of 0.1 to 0.3 .
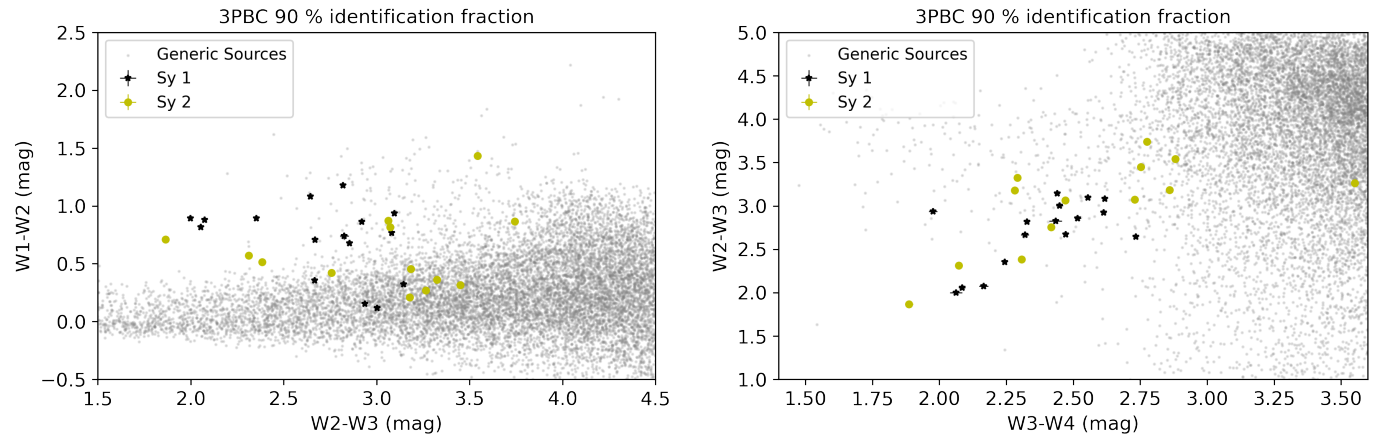

Fig. B.7. Subsample with $90 \%$ of Seyfert galaxies above $90 \%$ identification fraction in the 3PBC catalog. The color-coding is the same as in Figure B.1. 